\title{
Minimax-Based Selection, Adjustment and Disaggregation for Incompletely Specified Univariate Distributions
}

\begin{abstract}
A. J. Goldman
Institute for Basic Standards, National Bureau of Standards, Washington, D.C. 20234

(December 10, 1971)

This paper deals with making a nominal choice from a class of discrete univariate probability dis tributions about which one has "incomplete information" such as componentwise bounds, a component wise ranking, or both. In some cases an initial distribution is provided, to be "adjusted" so as to be com patible with the incomplete information. The first part of the analysis systematizes and unifies the contents of four earlier papers treating such problems using a minimax-error or minimax-adjustment approach. The second part applies the same approach to situations in which an "aggregate" of the desired distribution is stipulated, either exactly, or approximately by componentwise bounds. All problems discussed in the paper can be formulated as linear programs, but relatively explicit solution methods are sought; unresolved difficulties arising in this attempt are identified.
\end{abstract}

Key words: Linear programs; mathematical models; minimax estimation; operations research; probability distribution.

\section{Introduction}

In the mathematical modeling efforts associated with an operations research study, it may be necessary to attribute numerical values to the probabilities of the various possible outcomes of some chance event, even though the information at hand is not sufficient to determine this probability distribution uniquely. Under these circumstances, it seems useful to have a systematic and reproducible method for selecting some one distribution from among those compatible with the available data, preferably for use as a "base-point" or "nominal case" for a subsequent sensitivity analysis. ${ }^{1}$

The paper which follows is the fifth in a series exploring one approach to this problem area. As before, attention is confined to discrete univariate distributions ${ }^{2}$ such a distribution will be represented by a real $n$-vector $\mathbf{x}$ which is a probability vector, i.e., its components $x_{i}$ are nonnegative and sum to unity. The "incomplete information" about the "true" distribution is summarized as a constraint $\mathbf{x} \epsilon P$, where $P$ is a closed bounded subset of $n$-space.

By the minimax error selection problem for $P$, we mean the problem of choosing $\mathbf{x} \epsilon P$ to minimize

$$
F(\mathbf{x})=\max \left\{\max _{i}\left|x_{i}-y_{i}\right|: \mathbf{y} \epsilon P\right\}
$$

AMS Subject Classification: Primary 90B99, Secondary 62F99, 90C05.

' NBS colleague R. C. Sansing, to whom (along with M. H. Pearl) I am indebted for a careful reading of the paper and for numerous helpful suggestions, points out that such a method might also prove useful to Bayesian data analysts in choosing a prior distribution consistent with the partial information at hand.

${ }^{2}$ A bivariate application will be given in [5]. 
The corresponding weighted problem for a vector $\mathbf{w}$ of positive "weights" $\left\{w_{i}\right\}_{1}{ }^{n}$, is that of choosing $\mathbf{x} \epsilon P$ to minimize

$$
F(\mathbf{x} ; \mathbf{w})=\max \left\{\max _{i} w_{i}\left|x_{i}-y_{i}\right|: \mathbf{y} \epsilon P\right\}
$$

This corresponds to the situation in which errors in the attributed probabilities of some outcomes of the chance event are significantly more serious than errors in the attributed probabilities of other outcomes.

The analysis of such problems can be facilitated by noting that $F(\mathbf{x} ; \mathbf{w})$ admits the more explicit representation

$$
F(\mathbf{x} ; \mathbf{w})=\max _{i} \max \left\{w_{i}\left(M_{i}-x_{i}\right), w_{i}\left(x_{i}-m_{i}\right)\right\}
$$

in terms of the quantities

$$
\begin{gathered}
M_{i}=\max \left\{y_{i}: \mathbf{y} \epsilon P\right\}, \\
m_{i}=\min \left\{y_{i}: \mathbf{y} \epsilon P\right\} .
\end{gathered}
$$

This follows from the commutability of the three "maximum" operators in

$$
F(\mathbf{x} ; \mathbf{w})=\max _{\mathbf{y} \epsilon P} \max _{i} \max \left\{w_{i}\left(x_{i}-y_{i}\right), w_{i}\left(y_{i}-x_{i}\right)\right\} .
$$

In view of (1.3), the weighted selection problem can be rephrased as that of selecting a number $z$ and an $n$-vector $\mathbf{x}$ so as to minimize $z$, subject to constraints

$$
\begin{array}{ll}
z \geqslant w_{i}\left(M_{i}-x_{i}\right) & \text { (all } i), \\
z \geqslant w_{i}\left(x_{i}-m_{i}\right) & \text { (all } i), \\
\mathbf{x} \in P . &
\end{array}
$$

If $P$ is the solution-set of a finite family of linear equations (e.g., $\Sigma_{i} x_{i}=1$ ) and/or inequalities (e.g., $x_{i} \geqslant 0$ ), then this problem is a linear program once the values of $M_{i}$ and $m_{i}$ are known; moreover $\boldsymbol{M}_{i}$ and $m_{i}$ are themselves determinable as the extremal values of linear programs. All the particular cases described below can in fact be solved numerically by standard linear programming methods; however, we set a higher standard, expecting that the special form of these linear programs admits a solution algorithm which is more explicit (nearer to "closed-form"). The degree of explicitness demanded can best be understood by examining the previous papers $[1-4],{ }^{3}$ or the material in section 2 of the present paper.

The information expressed by the condition $\mathbf{x} \epsilon P$ may be accompanied by information (e.g., data on some past analogous situation) suggesting an estimate of the desired distribution. We assume that this estimate, if present, is given as a probability $n$-vector a, which is to be "adjusted as little as possible" to yield a distribution $\mathbf{x} \in P$. This leads to the minimax adjustment problem for $(\mathbf{a}, P)$, of choosing $\mathbf{x} \epsilon P$ to minimize

$$
G(\mathbf{x})=\max _{i}\left|x_{i}-a_{i}\right|
$$

Again there is a more general weighted problem, that of choosing $\mathbf{x} \in P$ to minimize

$$
G(\mathbf{x} ; \mathbf{w})=\max _{i} w_{i}\left|x_{i}-a_{i}\right| .
$$

Such a problem can in turn be rephrased as that of selecting a number $z$ and an $n$-vector $\mathbf{x}$ so as to minimize $z$, subject to the constraints

$$
z \geqslant w_{i}\left(x_{i}-a_{i}\right) \quad(\text { all } i),
$$

${ }^{3}$ Figures in brackets indicate the literature references at the end of this paper. 


$$
\begin{aligned}
& \left.z \geqslant w_{i}\left(a_{i}-x_{i}\right) \quad \text { (all } i\right), \\
& \mathbf{x} \boldsymbol{\epsilon} P .
\end{aligned}
$$

This too is a linear program when $P$ is the solution-set of a finite family of linear equations and/or inequalities; once again the cases to be discussed are accessible numerically to linear programming techniques, but once again we seek more explicit solution algorithms.

The present paper has three distinguishable purposes. The first is to provide a more unified and systematic account of the material in [1-4] than was apparent when that material was first worked out. The second objective is to invite the attention of colleagues to some desired generalizations which as yet have proven recalcitrant in terms of the "more explicit solution algorithm" criterion mentioned above; the analysis of these generalizations will be carried here to the point where the mathematical difficulty becomes explicit, in the hope of stimulating others to resolve these difficulties.

The third goal is to apply this general methodology in a new area: that of disaggregating a given or "approximately given" probability distribution. The hypothesized situation here is that probabilities have been observed or estimated, for the outcomes of the chance event in question, according to some relatively crude classification of these outcomes; the problem at hand is to attribute numerical probabilities to the outcomes as specified in some finer classification. In other words, probabilities are given for "super-events" $E_{j}$ each of which is a collection of mutually exclusive elementary events $E_{i j}$, and the aim is to specify the probabilities of the $E_{i j}$ 's "optimally" in the sense of the minimax criterion used here. Readers familiar with "real-world" data-analysis and modeling problems will readily recognize how such problems can arise.

The following material is organized as follows: section 2 contains descriptions of, and status reports on, several subproblems to which our main problems can be reduced; section 3 both unifies and summarizes the cases satisfactorily treated in the four earlier papers, and also shows how the as-yet-unresolved generalizations of these cases reduce to certain of the subproblems presented in section 2.

In section 4, we consider the "minimax error selection" and "minimax adjustment" problems for the "disaggregation situation" described above, assuming that the distribution to be disaggregated is known exactly. The unweighted versions of these problems, and a restricted class of weighted versions, are shown to be solvable. Section 5 establishes the analogous results for the case in which the "crude" distribution is specified only in terms of upper and lower bounds on its components.

One further technical point should be noted. The problems described above will all be reduced to the form: choose number $z$ and $n$-vector $\mathbf{x}$ to minimize $z$ subject to certain constraints. In general, the extremal solution is not unique; along with $z_{\min }$ one has a convex polyhedron of optimizing $\mathbf{x}$ 's rather than a single $\mathbf{x}$. A plausible "representative choice" from this polyhedron is the centroid of its vertices. The determination of this centroid is treated in some detail in section 5 of [1] and section 3 of [2], for the particular cases studied in those papers. These detailed treatments are regarded as adequate indications of how the analysis might go in other cases; thus the "centroid" approach to resolution of nonuniqueness is not taken up explicitly in [3] or [4] or the present paper.

\section{Subproblems}

In this section we describe several subproblems which will arise later, and report on their status as regards effective solution-methods. The reader may prefer to skip this section at first, coming back to it as the individual subproblems arise.

Problem I: Given $m$-vectors $\mathbf{A}$ and $\mathbf{B}$, and number $S$, find an $m$-vector $\mathbf{y}$ such that ${ }^{4}$

$$
\begin{gathered}
\mathbf{A} \leqslant \mathbf{y} \leqslant B, \\
\Sigma_{k} y_{k}=S .
\end{gathered}
$$

${ }^{4}$ Inequalities between vectors are to be interpreted componentwise. 
Obvious necessary conditions for a solution to exist are

$$
\begin{gathered}
\mathbf{A} \leqslant \mathbf{B}, \\
\Sigma_{k} A_{k} \leqslant S \leqslant \Sigma_{k} B_{k} .
\end{gathered}
$$

These conditions are also sufficient. For if they hold, then when $\mathbf{A}=\mathbf{B}$ we can set $\mathbf{y}=\mathbf{A}$ and satisfy (2.1) and (2.2). When $\mathbf{A} \neq \mathbf{B}$ we can define

$$
\Theta=\left[S-\Sigma_{k} A_{k}\right] /\left[\Sigma_{k} B_{k}-\Sigma_{k} A_{k}\right]
$$

so that $0 \leqslant \Theta \leqslant 1$ by virtue of (2.4), and then satisfy (2.1)-(2.2) by putting

$$
\mathbf{y}=(1-\theta) \mathbf{A}+\Theta \mathbf{B} \text {. }
$$

Problem II. Given $m$-vectors $\mathbf{A}$ and $\mathbf{B}$, and number $S$, find an $m$-vector $\mathbf{y}$ satisfying not only (2.1)(2.2), but also

$$
y_{1} \leqslant y_{2} \leqslant \ldots \leqslant y_{m} .
$$

Here it is convenient to define nondecreasing sequences $\left\{A_{k}{ }^{\prime}\right\}_{1}{ }^{m}$ and $\left\{B_{k}{ }^{\prime}\right\}_{1}{ }^{m}$, forming the components of respective vectors $\mathbf{A}^{\prime}$ and $\mathbf{B}^{\prime}$, by

$$
A_{k}{ }^{\prime}=\max _{j \leqslant k} A_{j}, \quad B_{k}{ }^{\prime}=\min _{j \geqslant k} B_{j} .
$$

Obvious necessary conditions for a solution to exist are

$$
\begin{gathered}
\left.\mathbf{A}^{\prime} \leqslant \mathbf{B}^{\prime} \quad \text { (i.e., } A_{i} \leqslant B_{j} \text { for } i \leqslant j\right), \\
\sum_{k} A_{k}^{\prime} \leqslant S \leqslant \Sigma_{k} B_{k}^{\prime}
\end{gathered}
$$

That these conditions are also sufficient follows from the same construction as above, applied to $\mathbf{A}^{\prime}$ and $\mathbf{B}^{\prime}$.

Problem III: Given $m$-vector $\mathbf{Z}$, positive $m$-vector $\mathbf{v}$, and number $S$, find the minimum value $z^{*}$ of $z$ such that $z \geqslant 0$ and

$$
\Sigma_{k} \max \left\{0, Z_{k}-z / v_{k}\right\} \leqslant S .
$$

An obvious necessary condition for the existence of a solution is

$$
S \geqslant 0 \text {. }
$$

That this condition is also sufficient is shown in section 2 of [4], where the following solution algorithm is justified subject to (2.10) as hypothesis.

First delete all $Z_{k} \leqslant 0$ from the problem; if no $Z_{k}$ are left then $z^{*}=0$. Suppose some are left; choose $Z_{m+1}=0$ and any $v_{m+1}>0$, and renumber so that

$$
v_{1} Z_{1} \geqslant v_{2} Z_{2} \geqslant \ldots \geqslant v_{m} Z_{m}>v_{m+1} Z_{m+1}=0 .
$$

The sequence $\left\{Z_{k}{ }^{*}\right\}_{1}{ }^{m+1}$ defined by

$$
Z_{k}^{*}=\Sigma_{1}{ }^{k} Z_{j}-v_{k} Z_{k} \Sigma_{1}{ }^{k}\left(1 / v_{j}\right)
$$

is calculable by the recursion

$$
Z_{k+1}^{*}=Z_{k}{ }^{*}+\left(v_{k} Z_{k}-v_{k+1} Z_{k+1}\right) \Sigma_{1}{ }^{k}\left(1 / v_{j}\right)
$$

and initial condition $Z_{1}^{*}=0$. Unless $\Sigma_{k} Z_{k}<S$ (in which case $z^{*}=0$ ), there is a unique $J \epsilon\{1,2, \ldots$, $m$ \} such that

$$
0=Z_{1}{ }^{*} \leqslant Z_{2}{ }^{*} \leqslant \ldots \leqslant Z_{J}{ }^{*}<S \leqslant Z_{J+1}{ }^{*} \leqslant \ldots \leqslant Z_{m+1} * \text {. }
$$


In terms of this $J$,

$$
z^{*}=\left[\Sigma_{1}{ }^{J} Z_{k}-S\right] / \Sigma_{1}{ }^{J}\left(1 / v_{k}\right)
$$

Problem IV: Given $m$-vector $\mathbf{Z}$, positive $m$-vector $\mathbf{v}$ and number $S$, find the minimum value $z^{*}$ of $z$ such that $z \geqslant 0$ and

$$
z-\Sigma_{k} \max \left\{0, Z_{k}-z / v_{k}\right\} \geqslant S .
$$

This problem, not taken up explicitly in the earlier papers, will now be solved. The left-hand side of (2.15) is a continuous increasing function of $z$, bounded neither above nor below. The problem therefore always has a solution, and that solution is $z^{*}=0$ if and only if the value of this function for $z=0$ is $\geqslant S$. From now on we assume the opposite case,

$$
S+\Sigma_{k} \max \left\{0, Z_{k}\right\}>0 ;
$$

in this case $z^{*}$ is the unique value of $z$ for which equality holds in (2.15).

Clearly all $Z_{k} \leqslant 0$ can be deleted from the problem. If none are left, then $z^{*}=S$; from now on we assume some left. Set $Z_{m+1}=0$, choose any $v_{m+1}>0$, and renumber so that

$$
v_{1} Z_{1} \geqslant v_{2} Z_{2} \geqslant \ldots \geqslant v_{m} Z_{m}>v_{m+1} Z_{m+1}=0
$$

Define a sequence $\left\{Z_{k}{ }^{*}\right\}_{1}{ }^{m+1}$ by

$$
Z_{k}^{*}=v_{k} Z_{k}\left[1+\Sigma_{1}{ }^{k}\left(1 / v_{j}\right)\right]-\Sigma_{1}{ }^{k} Z_{j}
$$

it can be calculated from the initial condition $Z_{1}{ }^{*}=v_{1} Z_{1}$ and the recursion

$$
Z_{k+1} *=Z_{k}^{*}+\left(v_{k+1} Z_{k+1}-v_{k} Z_{k}\right)\left[1+\Sigma_{1}{ }^{k}\left(1 / v_{j}\right)\right],
$$

which shows the sequence to be nonincreasing. Since (2.16) with strict inequality (and all $Z_{k}>0$ ) implies $S>Z_{m+1}{ }^{*}$, there is a least $K \epsilon\{1,2, \ldots m+1\}$ for which $S>Z_{K}{ }^{*}$.

If $z \leqslant v_{K} Z_{K}$, then

$$
\begin{aligned}
z-\Sigma_{k} \max \left\{0, Z_{k}-z / v_{k}\right\} & \leqslant z-\Sigma_{1}{ }^{K}\left(Z_{k}-z / v_{k}\right) \\
& =z\left[1+\Sigma_{1}{ }^{K}\left(1 / v_{j}\right)\right]-\Sigma_{1}{ }^{K} Z_{j} \\
& \leqslant v_{K} Z_{K}\left[1+\Sigma_{1}{ }^{K}\left(1 / v_{j}\right)\right]-\Sigma_{1}{ }^{K} Z_{j} \\
& =Z_{K}{ }^{*}<S,
\end{aligned}
$$

so that (2.15) is not satisfied. But if $K>1$ and $v_{K} Z_{K}<z \leqslant v_{K-1} Z_{K-1}$, then (2.15) becomes

$$
z-\Sigma_{1}{ }^{K-1}\left(Z_{k}-z / v_{k}\right) \geqslant S,
$$

which is equivalent to

$$
z \geqslant z^{*}=\left[\Sigma_{1}{ }^{K-1} Z_{k}+S\right] /\left[1+\Sigma_{1}{ }^{K-1}\left(1 / v_{k}\right)\right]
$$

The value of $z^{*}$ proposed in (2.19) is readily verified to satisfy $v_{K} Z_{K} \leqslant z^{*} \leqslant v_{K-1} Z_{K-1}$, and so is indeed the smallest $z \geqslant 0$ satisfying (2.15). Finally, if $K=1$ so that $S>Z_{1}{ }^{*}=v_{1} Z_{1}$, then $v_{1} Z_{1}<z$ turns (2.15) into $z \geqslant S$, so that

$$
z^{*}=S \quad(\text { for } K=1)
$$

Problem V: Given $m$-vector $\mathbf{Z}$, positive $m$-vector $\mathbf{v}$ and number $S$, find the minimum value $z^{*}$ of $z$ such that $z \geqslant 0$ and

$$
\Sigma_{k} \max \left\{0, \max _{j \leqslant k}\left(Z_{j}-z / v_{j}\right)\right\} \leqslant S .
$$


We do not presently have a satisfactory solution method for this problem, not even under the additional assumption

$$
0<Z_{1} \leqslant Z_{2} \leqslant \ldots \leqslant Z_{m}
$$

which will sometimes hold when the problem arises later. Note however that if $\mathbf{Z}$ and $\mathbf{v}$ are similarly ordered, in the sense that there exists a permutation $\pi$ of $\{1,2, \ldots, m\}$ for which

$$
\begin{gathered}
Z_{\pi(1)} \leqslant Z_{\pi(2)} \leqslant \ldots \leqslant Z_{\pi(m),}, \\
v_{\pi(1)} \leqslant v_{\pi(2)} \leqslant \ldots \leqslant v_{\pi(m)},
\end{gathered}
$$

both hold, then the problem can be handled by previous methods. For in this case, for each $k \epsilon\{1,2, \ldots, m\}$ there is a $p(k) \epsilon\{1,2, \ldots, k\}$ such that

$$
Z_{p(k)}=\max _{j \leqslant k} Z_{j}, \quad v_{p(k)}=\max _{j \leqslant k} v_{j},
$$

so that for $z \geqslant 0(2.21)$ is equivalent to

$$
\Sigma_{k} \max \left\{0, Z_{p(k)}-z / v_{p(k)}\right\} \leqslant S
$$

and we are back to an instance of Problem III. This comment will, of course, in particular apply whenever either all $Z_{k}$ are equal or all $v_{k}$ are equal.

Problem VI: Given $m$-vector $\mathbf{Z}$, positive $m$-vector $\mathbf{v}$ and number $S$, find the minimum value $z^{* *}$ of $z$ such that $z \geqslant 0$ and

$$
\Sigma_{k} \min _{j \geqslant k}\left(Z_{j}+z / v_{j}\right) \geqslant S
$$

Here too, a solution method is presently lacking in the general case. Suppose, however, that $\mathbf{Z}$ and $\mathbf{v}$ are oppositely ordered, in the sense that there exists a permutation $\pi$ of $\{1,2, \ldots, m\}$ for which

$$
\begin{gathered}
Z_{\pi(1)} \leqslant Z_{\pi(2)} \leqslant \ldots \leqslant Z_{\pi(m)}, \\
v_{\pi(1)} \geqslant v_{\pi(2)} \geqslant \ldots \geqslant v_{\pi(m)}
\end{gathered}
$$

both hold. Then for each $k \epsilon\{1,2, \ldots, m\}$, there is a $p(k) \epsilon\{k, k+1, \ldots, m\}$ such that

$$
Z_{p(k)}=\min _{j \geqslant k} Z_{j}, \quad v_{p(k)}=\max _{j \geqq k} v_{j} .
$$

Then for $z \geqslant 0,(2.23)$ is equivalent to

$$
\Sigma_{k}\left(Z_{\mu(k)}+z / v_{\mu(k)}\right) \geqslant S,
$$

so that in this case $z^{* *}$ is given by

$$
z^{* *}=\max \left\{0,\left[S-\Sigma_{k} Z_{p(k)}\right] / \Sigma_{k}\left(1 / v_{p(k)}\right)\right\} .
$$

This of course applies, in particular, if all $Z_{k}$ are equal or if all $v_{k}$ are equal.

Problem VII: Given $m$-vectors $\mathbf{Y}$ and $\mathbf{Z}$, positive $m$-vector $\mathbf{v}$ and number $S$, find the maximum value $z^{*}$ of $z$ such that $z \geqslant 0$ and

$$
\Sigma_{k} \max \left\{Y_{k}, \max _{j \leqslant k}\left(Z_{j}-z / v_{j}\right)\right\} \leqslant S .
$$

This has Problem V as a special case $(\mathbf{Y}=\mathbf{O})$, and so also at present lacks a satisfactory solution method except when $\mathbf{Z}$ and $\mathbf{v}$ are similarly ordered.

Resolution of the three Problems (V, VI, VII), presented so far as in the "unsolved" category, 
would (as demonstrated later) yield solution methods for the "weighted" versions of the problems whose unweighted cases were solved in [2-4].

We turn now to problems associated with the new "disaggregation" considerations taken up in sections 4 and 5 . These involve a partition of $\{1,2, \ldots, n\}$ into nonempty sets $\left\{S_{j}\right\}_{1}{ }^{m}$. For any $n$-vector $\mathbf{x}$, and any subset $T$ of $\{1,2, \ldots, n\}$, we set

$$
\mathbf{x}(T)=\Sigma\left\{x_{i}: i \epsilon T\right\}=\Sigma_{i \epsilon T} x_{i} .
$$

Problem VIII: Given $n$-vectors $\mathbf{A}$ and $\mathbf{B}, m$-vectors $\mathbf{s}^{-}$, and $\mathbf{s}^{+}$, and number $S$, find an $n$-vector $\mathbf{x}$ such that

$$
\begin{gathered}
\mathbf{A} \leqslant \mathbf{x} \leqslant \mathbf{B}, \\
\sum_{i} x_{i}=S, \\
s_{j}^{-} \leqslant \mathbf{x}\left(S_{j}\right) \leqslant s_{j}^{+} \quad \text { for } 1 \leqslant j \leqslant m .
\end{gathered}
$$

An obvious necessary condition for a solution, in view of (2.27), is

$$
\mathbf{A} \leqslant \mathbf{B} .
$$

Another is

$$
\max \left\{s_{j}^{-}, \mathbf{A}\left(S_{j}\right)\right\} \leqslant \min \left\{s_{j}{ }^{+}, \mathbf{B}\left(S_{j}\right)\right\} \quad(1 \leqslant j \leqslant m),
$$

while a third is

$$
\Sigma_{j} \max \left\{s_{j}^{-}, \mathbf{A}\left(S_{j}\right)\right\} \leqslant S \leqslant \Sigma_{j} \min \left\{s_{j}{ }^{+}, \mathbf{B}\left(S_{j}\right)\right\} .
$$

We now give a constructive proof that this trio of necessary conditions is also sufficient. Suppose the conditions hold. By (2.31) and (2.32), plus our analysis of Problem I, there is an $m$-vector $\mathbf{y}$ such that

$$
\begin{gathered}
\max \left\{s_{j}^{-}, \mathbf{A}\left(S_{j}\right)\right\} \leqslant y_{j} \leqslant \min \left\{s_{j}{ }^{+}, \mathbf{B}\left(S_{j}\right)\right\} \quad(1 \leqslant j \leqslant m), \\
\Sigma y_{j}=S .
\end{gathered}
$$

Since $\mathbf{A}\left(S_{j}\right) \leqslant y_{j} \leqslant \mathbf{B}\left(S_{j}\right)$, we can write

$$
y_{j}=\left(1-\Theta_{j}\right) \mathbf{A}\left(S_{j}\right)+\Theta_{j} \mathbf{B}\left(S_{j}\right)
$$

with $\Theta_{j} \in[0,1]$. (If $\mathbf{A}\left(S_{j}\right)=\mathbf{B}\left(S_{j}\right)$, then $\Theta_{j}$ is arbitrary.)

Now define $n$-vector $\mathbf{x}$ by

$$
x_{i}=\left(1-\Theta_{j}\right) A_{i}+\Theta_{j} B_{i} \quad\left(i \epsilon S_{j}\right)
$$

then (2.27) is satisfied since $\mathbf{A} \leqslant \mathbf{B}$ and $\Theta_{j} \in[0,1]$. Since $\mathbf{x}\left(S_{j}\right)=y_{j},(2.28)$ and (2.29) follow from (2.34) and (2.33), respectively.

Problem IX: Given $m$-vector $\mathbf{Y}$, positive $n$-vector $\mathbf{w}, n$-vector $\mathbf{Z}$ and number $S$, find the minimum value $z^{*}$ of $z$ such that $z \geqslant 0$ and

$$
\Sigma_{j} \max \left\{Y_{j}, \Sigma_{i \epsilon S_{j}} \max \left\{0, Z_{i}-z / w_{i}\right\}\right\} \leqslant S .
$$

Note first that if $Z_{i} \leqslant 0$, then the associated summand in (2.36) vanishes. Thus we may assume all $Z_{i}>0$. Next, an obvious necessary condition for a solution to exist is

$$
\Sigma_{j} \max \left\{Y_{j}, 0\right\} \leqslant S ;
$$

this will be assumed in what follows. Finally, it will be assumed that

$$
\Sigma_{j} \max \left\{Y_{j}, \Sigma_{i \in S} Z_{i}\right\}>S
$$


since in the contrary case we would have $z^{*}=0$.

Choose $Z_{o}=0$ and arbitrary $w_{0}>0$; renumber if necessary so that

$$
0=w_{0} Z_{o}<w_{1} Z_{1} \leqslant w_{2} Z_{2} \leqslant \ldots \leqslant w_{n} Z_{n} .
$$

By (2.37) and (2.38), we have $0<z^{*}<w_{n} Z_{n}$.

For $k \in\{1,2, \ldots, n\}$, set

$$
\begin{gathered}
S_{j k}=\left\{i: i \epsilon S_{j}, i \geqslant k\right\}=S_{j} \cap\{k, k+1, \ldots, n\}, \\
Z_{j k}=\Sigma\left\{Z_{i}: i \epsilon S_{j k}\right\}=\mathbf{Z}\left(S_{j k}\right), \\
1 / v_{j k}=\Sigma\left\{1 / w_{i}: i \epsilon S_{j k}\right\} .
\end{gathered}
$$

Then for $z \epsilon\left(w_{k-1} Z_{k-1}, w_{k} Z_{k}\right]$, we have

$$
\left[i \epsilon S_{j} \text { and } z \leqslant w_{i} Z_{i}\right] \quad \text { iff } i \epsilon S_{j k},
$$

and thus

$$
\Sigma_{i \epsilon S_{j}} \max \left\{0, Z_{i}-z / w_{i}\right\}=Z_{j k}-z / v_{j k} .
$$

Thus, $z \epsilon\left(w_{k-1} Z_{k-1}, w_{k} Z_{k}\right]$ satisfies (2.36) if and only if

$$
\Sigma_{j} \max \left\{Y_{j}, Z_{j k}-z / v_{j k}\right\} \leqslant S,
$$

which can be rewritten

$$
\Sigma_{j} \max \left\{0,\left(Z_{j k}-Y_{j}\right)-z / v_{j k}\right\} \leqslant S-\Sigma_{j} Y_{j} .
$$

This in turn can be true for some $z \epsilon\left(w_{k-1} Z_{k-1}, w_{k} Z_{k}\right]$, if and only if it is true for $z=w_{k} Z_{k}$, i.e.

$$
\Sigma_{j} \max \left\{0,\left(Z_{j k}-Y_{j}\right)-w_{k} Z_{k} / v_{j k}\right\} \leqslant S-\Sigma_{j} Y_{j} .
$$

These observations yield the following procedure for finding $z^{*}$. First, try $k=1,2, \ldots$, until reaching a first value for which (2.42) is satisfied. Second, with this value of $k$ solve the instance (2.41) of Problem III. (We have not considered how the information $z^{*} \epsilon\left(w_{k-1} Z_{k-1}, w_{k} Z_{k}\right]$ might be used to streamline the second step.)

Problem X: Given $m$-vector $\mathbf{Y}, n$-vector $\mathbf{Z}$, positive $n$-vector $\mathbf{w}$, number $S$, and for each $j \in\{1,2, \ldots$, $m\}$ an enumeration of the $\sigma(j)$ members of $S_{j}$; write $w_{i}=w_{r}{ }^{j}$ and $Z_{i}=Z_{r}{ }^{j}$ if $i$ is the $r$ th member of $S_{j}$; find the least value $z^{*}$ of $z$ such that $z \geqslant 0$ and

$$
\Sigma_{j} \max \left\{Y_{j}, \Sigma_{r=1}^{\sigma(j)} \max \left\{0, \max _{R \leqslant r}\left(Z_{R}^{j}-z / w_{R}^{j}\right)\right\}\right\} \leqslant S .
$$

We presently lack a satisfactory solution method for this problem.

Problem XI: In the same setting as for Problem X, find the least value $z^{* *}$ of $z$ such that $z \geqslant 0$ and

$$
\Sigma_{j} \min \left\{Y_{j}, \min _{R \geqslant r}\left(Z_{r^{j}}+z / w_{r}{ }^{j}\right)\right\} \geqslant S .
$$

Here too, we still lack a satisfactory algorithm.

\section{Previous Work and Unsolved Cases}

\subsection{Componentwise Bounds}

A natural kind of "incomplete information," about a probability distribuiion arising in a practical context, consists of lower and upper bounds on the components of the probability $n$-vector 
representing the distribution. If $\mathbf{L}$ and $\mathbf{U}$ denote the respective vectors of lower and upper bounds then the associated constraint set is the convex polyhedron

$$
P=\left\{\mathbf{x}: \mathbf{x} \geqslant \mathbf{O}, \Sigma_{i} x_{i}=1, \mathbf{L} \leqslant \mathbf{x} \leqslant \mathbf{U}\right\} .
$$

Replacing $\mathbf{L}$ by $\max \{\mathbf{O}, \mathbf{L}\}$ and $\mathbf{U}$ by $\min \{\mathbf{U}, \mathbf{l}\}$ if necessary, we can assume without loss of generality that $\mathbf{L} \geqslant 0$ and $\mathbf{U} \leqslant \mathbf{1}$. By the results for Problem $I$ in section 2 , the necessary and sufficient conditions for $P$ to be nonempty are

$$
\begin{gathered}
\mathbf{L} \leqslant \mathbf{U}, \\
\Sigma_{i} L_{i} \leqslant 1 \leqslant \Sigma_{i} U_{i} ;
\end{gathered}
$$

these will now be assumed.

In [1], the weighted minimax error selection problem for this set $P$ is solved. The following treatment, however, fits better with the present paper's framework. Observe that the problem can be transformed to that of choosing a number $z$ and an $n$-vector $\mathbf{x}$ to minimize $z$ subject to the constraints (1.6)-(1.8). In the present case, these constraints can be rewritten

$$
\begin{gathered}
\max \left\{L_{i}, M_{i}-z / w_{i}\right\} \leqslant x_{i} \leqslant \min \left\{U_{i}, m_{i}+z / w_{i}\right\} \quad(\text { all } i), \\
\Sigma_{i} x_{i}=1 .
\end{gathered}
$$

For any fixed $z$, our results for Problem I show (constructively) that an associated $\mathbf{x}$ exists if and only if

$$
\begin{gathered}
\left.\max \left\{L_{i}, M_{i}-z / w_{i}\right\} \leqslant \min \left\{U_{i}, m_{i}+z / w_{i}\right\} \quad \text { (all } i\right), \\
\Sigma_{i} \max \left\{L_{i}, M_{i}-z / w_{i}\right\} \leqslant 1, \\
\Sigma_{i} \min \left\{U_{i}, m_{i}+z / w_{i}\right\} \geqslant 1 .
\end{gathered}
$$

The redundant constraint $z \geqslant 0$ can be imposed if desired.

Now the left-hand sides of (3.1.4) and (3.1.5) are nonincreasing in $z$, while the right-hand side of (3.1.4) and the left-hand side of (3.1.6) are nondecreasing. It follows that the desired minimum value of $z$ can be written

$$
z_{\min }=\max \left\{z^{\circ}, z^{*}, z^{* *}\right\}
$$

where we put

$$
\begin{gathered}
z^{\circ}=\operatorname{minimum} z \text { satisfying }(3.1 .4) \\
z^{*}=\text { minimum nonnegative } z \text { satisfying (3.1.5), } \\
z^{* *}=\text { minimum nonnegative } z \text { satisfying (3.1.6). }
\end{gathered}
$$

In view of $(3.1 .2), z^{\circ}$ is well-defined, and is readily evaluated as

$$
z^{\circ}=\max _{i} \max \left\{w_{i}\left(L_{i}-m_{i}\right), w_{i}\left(M_{i}-U_{i}\right), w_{i}\left(M_{i}-m_{i}\right) / 2\right\} .
$$

Since $L_{i} \leqslant m_{i} \leqslant M_{i} \leqslant U_{i}$, this reduces to

$$
z^{\circ}=1 / 2 \max _{i}\left\{w_{i}\left(M_{i}-m_{i}\right)\right\} .
$$

By writing (3.1.5) as

$$
\Sigma_{i} \max \left\{0,\left(M_{i}-L_{i}\right)-z / w_{i}\right\} \leqslant 1-\Sigma_{i} L_{i},
$$

we see that the determination of $z^{*}$ is an instance of Problem III in section 2, obeying the feasibility condition (2.10) by virtue of the left-hand half of (3.1.3). 
Similarly, by rewriting (3.1.6) as

$$
\Sigma_{i} \max \left\{0,\left(U_{i}-m_{i}\right)-z / w_{i}\right\} \leqslant \Sigma_{i} U_{i}-1,
$$

we see that the determination of $z^{* *}$ is also an instance of Problem III in section 2, obeying the feasibility condition (2.10) by virtue of the right-hand half of (3.1.3).

[Some calculation can be avoided on the basis of the following additional analysis. By (3.1.8), $-z^{\circ} / w_{i} \leqslant-\left(M_{i}-m_{i}\right) / 2$, and so

$$
\begin{gathered}
\Sigma_{i} \max \left\{0,\left(M_{i}-L_{i}\right)-z^{\circ} / w_{i}\right\} \leqslant \Sigma_{i} \max \left\{0,\left(M_{i}+m_{i}\right) / 2-L_{i}\right\} \\
=\Sigma_{i}\left(M_{i}+m_{i}\right) / 2-\Sigma_{i} L_{i}, \\
\Sigma_{i} \max \left\{0,\left(U_{i}-m_{i}\right)-z^{\circ} / w_{i}\right\} \leqslant \Sigma_{i} \max \left\{0, U_{i}-\left(M_{i}+m_{i}\right) 2\right\} \\
=\Sigma_{i} U_{i}-\Sigma_{i}\left(M_{i}+m_{i}\right) / 2 .
\end{gathered}
$$

Comparing these relations with (3.1.9) and (3.1.10) respectively, we find that

$$
\begin{aligned}
& z^{\circ} \geqslant z^{*} \quad \text { if } \Sigma_{i}\left(M_{i}+m_{i}\right) \leqslant 2, \\
& z^{\circ} \geqslant z^{* *} \quad \text { if } \Sigma_{i}\left(M_{i}+m_{i}\right) \geqslant 2 .
\end{aligned}
$$

Thus a preliminary evaluation of $\Sigma_{i}\left(M_{i}+m_{i}\right)$ will render unnecessary the determination of either $z^{*}$ or $z^{* *}$.]

Since determination of all three quantities compared in (3.1.7) can be regarded as "solved" problems, the same can be said for the desired minimization of $z$. There is only one gap, namely the explicit determination - for the set $P$ specified in (3.1.1) - of the quantities $M_{i}$ and $m_{i}$, defined by (1.4) and (1.5), which figure in determining $z^{\circ}, z^{*}$ and $z^{* *}$.

For this purpose, note that for any $i$ the condition $\mathbf{x} \epsilon P$ can be rewritten

$$
\begin{gathered}
L_{i} \leqslant x_{i} \leqslant U_{i}, \\
L_{j} \leqslant x_{j} \leqslant U_{j} \quad(\text { all } j \neq i), \\
\Sigma_{j \neq i} x_{j}=1-x_{i} .
\end{gathered}
$$

For any fixed $x_{i}$, the existence of an $(n-1)$-vector $\left(x_{1}, \ldots, x_{i-1}, x_{i+1}, \ldots, x_{n}\right)$ satisfying the last two conditions is shown by the results for Problem I (in sec. 2) to have the necessary and sufficient conditions $L_{j} \leqslant U_{j}$ for all $j \neq i$ (this is guaranteed by (3.1.2)) and

$$
\Sigma_{j \neq i} L_{j} \leqslant 1-x_{i} \leqslant \Sigma_{j \neq i} U_{j}
$$

Combining (3.1.12) and (3.1.15), we find that

$$
\begin{aligned}
& M_{i}=\min \left\{U_{i}, 1-\Sigma_{j \neq i} L_{j}\right\}, \\
& m_{i}=\max \left\{L_{i}, 1-\Sigma_{j \neq i} U_{j}\right\} .
\end{aligned}
$$

This completes the analysis of the weighted minimax error selection problem, in the case where "incomplete information" is expressed as "componentwise bounds," so that the constraint set $P$ is given by (3.1.1).

For this same case, we turn now to the minimax adjustment problem. The weighted version of this problem for $(\mathbf{a}, P)$ - for any probability $n$-vector $\mathbf{a}$-is solved in section 3 of [4], using a treatment summarized below.

We know the problem can be transformed to that of choosing a number $z$ and an $n$-vector $\mathbf{x}$ to minimize $z$ subject to the constraints (1.10)-(1.12), which in the present case can be rewritten

$$
\begin{gathered}
\left.\max \left\{L_{i}, a_{i}-z / w_{i}\right\} \leqslant x_{i} \leqslant \min \left\{U_{i}, a_{i}+z / w_{i}\right\} \quad \text { (all } i\right), \\
\Sigma_{i} x_{i}=1 .
\end{gathered}
$$


For any fixed $z$, our results for Problem I show (constructively) that an associated $\mathbf{x}$ exists if and only if

$$
\begin{gathered}
\left.\max \left\{L_{i}, a_{i}-z / w_{i}\right\} \leqslant \min \left\{U_{i}, a_{i}+z / w_{i}\right\} \quad \text { (all } i\right), \\
\Sigma_{i} \max \left\{L_{i}, a_{i}-z / w_{i}\right\} \leqslant 1 \\
\Sigma_{i} \min \left\{U_{i}, a_{i}+z / w_{i}\right\} \geqslant 1
\end{gathered}
$$

The redundant constraint $z \geqslant 0$ can be imposed if desired.

As before, we can express the desired minimum value of $z$ as

$$
z_{\min }=\max \left\{z^{\circ}, z^{*}, z^{* *}\right\}
$$

where now

$$
\begin{gathered}
z^{\circ}=\operatorname{minimum} z \text { satisfying }(3.1 .18), \\
z^{*}=\text { minimum nonnegative } z \text { satisfying (3.1.19), } \\
z^{* *}=\text { minimum nonnegative } z \text { satisfying (3.1.20). }
\end{gathered}
$$

In view of (3.1.2), $z^{\circ}$ is well-defined, and is readily evaluated as

$$
z^{\circ}=\max _{i} \max \left\{w_{i}\left(L_{i}-a_{i}\right), w_{i}\left(a_{i}-U_{i}\right)\right\} .
$$

By writing (3.1.19) and (3.1.20) as

$$
\begin{aligned}
& \Sigma_{i} \max \left\{0,\left(a_{i}-L_{i}\right)-z / w_{i}\right\} \leqslant 1-\Sigma_{i} L_{i}, \\
& \Sigma_{i} \max \left\{0,\left(U_{i}-a_{i}\right)-z / w_{i}\right\} \leqslant \Sigma_{i} U_{i}-1,
\end{aligned}
$$

we see that determining $z^{*}$ and $z^{* *}$ are instances of Problem III in section 2, with satisfaction of the feasibility condition (2.10) ensured by (3.1.3). Thus the problem of minimizing $z$ can be regarded as solved.

\subsection{Componentwise Ranking}

A second natural kind of "incomplete information," concerning a probability distribution arising in a practical context, would be a ranking of the probabilities of the various outcomes of the associated chance event. This translates into a componentwise ranking for the probability $n$-vector representing the distribution. Thus our constraint set here is given by

$$
P=\left\{\mathbf{x}: 0 \leqslant x_{1} \leqslant x_{2} \leqslant \ldots \leqslant x_{n}, \Sigma x_{i}=1\right\}
$$

and is nonempty. As noted in section 2 of [2], it is easily verified that the quantities $M_{i}$ and $m_{i}$ of (1.4)(1.5) are given in this case by

$$
\begin{gathered}
M_{i}=1 /(n+1-i) \\
m_{i}=0 \\
m_{n}=1 / n .
\end{gathered}
$$

The weighted minimax error selection problem becomes that of choosing number $z$ and $n$-vector $\mathbf{x}$ to minimize $z$ subject to constraints (1.6)-(1.8), which here become ${ }^{5}$

$$
\max \left\{0, M_{1}-z / w_{1}\right\} \leqslant x_{1} \leqslant z / w_{1},
$$

${ }^{5}$ We assume $n>1$ throughout. 


$$
\begin{gathered}
M_{i}-z / w_{i} \leqslant x_{i} \leqslant z / w_{i} \quad(1<i<n), \\
M_{n}-z / w_{n} \leqslant x_{n} \leqslant 1 / n+z / w_{n}, \\
\sum_{i} x_{i}=1, \\
x_{1} \leqslant x_{2} \leqslant \ldots \leqslant x_{n} .
\end{gathered}
$$

Let us dispose first of the trivial case $n=2$, when the constraints (3.2.5)-(3.2.9) become

$$
\begin{gathered}
\max \left\{0, \frac{1}{2}-z / w_{1}\right\} \leqslant x_{1} \leqslant z / w_{1}, \\
1-z / w_{2} \leqslant x_{2} \leqslant \frac{1}{2}+z / w_{2}, \\
x_{1}+x_{2}=1 \\
x_{1} \leqslant x_{2} .
\end{gathered}
$$

These can be rewritten, in terms of $x_{1}$ alone, as

$$
\max \left\{0, \frac{1}{2}-z / w_{1}, \frac{1}{2}-z / w_{2}\right\} \leqslant x_{1} \leqslant \min \left\{\frac{1}{2}, z / w_{1}, z / w_{2}\right\}
$$

which for $z \geqslant 0$ has a solution $x_{1}$ if and only if

$$
\max \left\{\frac{1}{2}-z / w_{1}, \frac{1}{2}-z / w_{2}\right\} \leqslant \min \left\{z / w_{1}, z / w_{2}\right\} .
$$

The smallest $z \geqslant 0$ satisfying this condition is

$$
z_{\min }=\max \left\{w_{1} / 4, w_{2} / 4,\left[2\left(1 / w_{1}+1 / w_{2}\right)\right]^{-1}\right\},
$$

which can be simplified to

$$
z_{\min }=(1 / 4) \max \left\{w_{1}, w_{2}\right\} .
$$

For $w_{1} \geqslant w_{2},(3.2 .14)$ and (3.2.10) yield $x_{1}=1 / 4$ when $z=z_{\min }$, while for $w_{1} \leqslant w_{2},(3.2 .14)$ and (3.2.11) yield $x_{2}=3 / 4$. Thus in either case the "optimal" $\mathbf{x}$ of the linear program is

$$
\mathbf{x}=(1 / 4,3 / 4) \quad(n=2) .
$$

Now we assume $n>2$. By the results for Problem II in section 2, for any fixed $z \geqslant 0$ the constraints (3.2.5)-(3.2.9) admit a solution $n$-vector $\mathbf{x}$ if and only if

$$
\begin{gathered}
1 /(n+1-i)-z / w_{i} \leqslant z / w_{j} \quad(1 \leqslant i \leqslant j<n), \\
1 /(n+1-i)-z / w_{i} \leqslant 1 / n+z / w_{n} \quad(1 \leqslant i \leqslant n), \\
\sum_{i} \max \left\{0, \max _{j \leqslant i}\left[1 /(n+1-j)-z / w_{j}\right]\right\} \leqslant 1, \\
\left(1 / n+z / w_{n}\right)+\sum_{i<n} \min \left[1 / n+z / w_{n}, \min _{i \leqslant j<n}\left(z / w_{j}\right)\right] \geqslant 1 .
\end{gathered}
$$

As before, the desired minimum value of $z$ can be written

$$
z_{\min }=\max \left\{z^{\circ}, z^{*}, z^{* *}\right\}
$$

where now

$$
z^{\circ}=\text { minimum value of } z \text { satisfying (3.2.15)-(3.2.16), }
$$$$
z^{*}=\text { minimum nonnegative value of } z \text { satisfying (3.2.17), }
$$$$
z^{* *}=\text { minimum nonnegative value of } z \text { satisfying (3.2.18). }
$$ 
It is easily verified that

$$
\begin{gathered}
z^{\circ}=\max \left\{\max _{i \leqslant j<n} 1 /\left[(n+1-i)\left(1 / w_{i}+1 / w_{j}\right)\right],\right. \\
\left.\max _{i}(i-1) /\left[n(n+1-i)\left(1 / w_{i}+1 / w_{n}\right)\right]\right\} .
\end{gathered}
$$

From this, we have

$$
z^{\circ} / w_{n} \geqslant(n-1) / 2 n, z^{\circ} / w_{j} \geqslant 1 / 2(n+1-j) \quad(j<n),
$$

so that

$$
\begin{gathered}
\left(1 / n+z^{\circ} / w_{n}\right)+\sum_{i<n} \min \left[1 / n+z^{\circ} / w_{n}, \min _{i \leqslant j<n}\left(z^{\circ} / w_{j}\right)\right] \\
\geqslant(n+1) / 2 n+\sum_{i<n} \min \left[(n+1) / 2 n, 1 / 2 \min _{i \leqslant j<n}(n+1-j)^{-1}\right] \\
=(n+1) / 2 n+\sum_{i<n} \min [(n+1) / 2 n, 1 / 2(n+1-i)] \\
=(n+1) / 2 n+\Sigma_{i<n} 1 / 2(n+1-i) \\
=\frac{1}{2}[(1+1 / n)+(1 / 2+1 / 3+\ldots+1 / n)]>1 ;
\end{gathered}
$$

comparison with (3.2.18) shows that $z^{\circ} \geqslant z^{* *}$.

The determination of $z^{* *}$, though not really necessary in view of the result in the previous paragraph, will be taken up next. Define $n$-vector $\mathbf{u}$ by

$$
\begin{gathered}
u_{n}=1 / w_{n}>0, \\
u_{n-i}=1 / \max \left\{w_{j}: i \leqslant j<n\right\} \quad(1 \leqslant i<n),
\end{gathered}
$$

so that

$$
u_{1} \geqslant u_{2} \geqslant \ldots \geqslant u_{n-1}>0
$$

and the condition (3.2.18) which defines $z^{* *}$ (together with $z^{* *} \geqslant 0$ ) is equivalent to

$$
\left(1 / n+z u_{n}\right)+\Sigma_{i<n} \min \left[1 / n+z u_{n}, z u_{i}\right] \geqslant 1 .
$$

We rewrite this last condition as

$$
z \Sigma_{i} u_{1}-\Sigma_{i<n} \max \left\{z\left(u_{i}-u_{n}\right), 1 / n\right\} \geqslant 0 .
$$

CASE 1. Suppose $u_{n} \geqslant u_{1}$, or equivalently, $w_{n} \leqslant \max \left\{w_{i}: i<n\right\}$. Then $u_{n} \geqslant u_{i}$ for $1 \leqslant i<n$. In view of the constraint $z \geqslant 0$ imposed on $z^{* *},(3.2 .21)$ becomes

$$
z \Sigma_{i} u_{i}-(n-1) / n \geqslant 0,
$$

leading to

$$
z^{* *}=(n-1) / n \Sigma_{i} u_{i} \quad\left(\text { if } u_{n} \geqslant u_{1}\right)
$$

CASE 2. If CASE 1 does not hold, there is a largest $J \epsilon\{1,2, \ldots, n-1\}$ such that $u_{J}>u_{n}$. Then (3.2.21) becomes

$$
z \Sigma_{i} u_{i}-(n-J-1) / n-\Sigma_{1}{ }^{J} \max \left\{z\left(u_{i}-u_{n}\right), 1 / n\right\} \geqslant 0,
$$

which we rewrite as

$$
\left.z\left\{\Sigma_{J+1}{ }^{n} u_{i}+J u_{n}\right\}-\Sigma_{1}{ }^{J} \max \left\{0,1 / n-z\left(u_{i}-u_{n}\right)\right\} \geqslant n-J-1\right) / n .
$$

That we now have an instance of Problem IV solved in section 2, can be seen by setting 


$$
\begin{aligned}
& T=\Sigma_{J+1}{ }^{n} u_{i}+J u_{n}, \\
& S=(n-J-1) / n T \\
& Z_{k}=1 / n T \quad(1 \leqslant k \leqslant J), \\
& v_{k}=T /\left(u_{k}-u_{n}\right) \quad(1 \leqslant k \leqslant J) .
\end{aligned}
$$

Turning now to (3.2.17) we see that determining $z^{*}$ is an instance of Problem $\mathrm{V}$ in section 2, for which a solution method is currently lacking. This then is the present bottleneck to solving the general weighted minimax error selection problem, for the case in which the "incomplete information" is represented by a componentwise ranking constraint on $\mathbf{x}$.

It $i s$, however, possible to determine $z_{\min }$ under the special hypothesis

$$
w_{n}=\max _{i} w_{i} .
$$

In this case we have for $i \leqslant j<n$,

$$
1 /(n+1-i)\left(1 / w_{i}+1 / w_{j}\right) \leqslant w_{n} / 2(n+1-i) \leqslant w_{n} / 4 \leqslant(n-1) w_{n} / 2 n,
$$

and also

$$
\max _{i}(i-1) /(n+1-i)\left(1 / w_{i}+1 / w_{n}\right)=(n-1) w_{n} / 2,
$$

so that (3.2.19) gives

$$
z^{\circ}=(n-1) w_{n} / 2 n,
$$

implying

$$
\begin{gathered}
\Sigma_{i} \max \left\{0, \max _{j \leqslant i}\left[1 /(n+1-j)-z^{\circ} / w_{j}\right]\right\} \\
\leqslant \Sigma_{i} \max \left\{0, \max _{j \leqslant i}[1 /(n+1-j)-(n-1) / 2 n]\right\} \\
=\Sigma_{i} \max \{0,1 /(n+1-i)-(n-1) / 2 n\} \\
=\Sigma_{j} \max \{0,1 / j-(n-1) / 2 n\}=\Sigma\{1 / j-(n-1) / 2 n: 1 \leqslant j \leqslant 2+2 /(n-1)\} .
\end{gathered}
$$

Considering separately the situations $n=3$ and $n \geqslant 4$, we find that the above expression equals ( $1 / 2$ $+1 / n) \leqslant 1$, and comparison with (3.2.17) yields $z^{\circ} \geqslant z^{*}$. Since it has already been established that $z^{\circ} \geqslant z^{* *}$, when (3.2.24) holds we have

$$
z_{\min }=z^{\circ}=\frac{1}{2}(1-1 / n) w_{n} .
$$

With this value of $z,(3.2 .7)$ and (3.2.2) imply that each optimal $\mathbf{x}$ has

$$
x_{n}=(n+1) / 2 n,
$$

"matching" (3.2.14a). These results agree with those obtained less systematically, for the unweighted case, in the earlier paper [2].

This completes the discussion of the "componentwise ranking" version of the mimimax error selection problem. We shift now to the same version of the minimax adjustment problem. The weighted case is that of choosing number $z$ and $n$-vector $\mathbf{x}$ to minimize $z$ subject to the constraints (1.10)-(1.12), which here become

$$
\begin{gathered}
a_{i}-z / w_{i} \leqslant x_{i} \leqslant a_{i}+z / w_{i} \quad(\text { all } i), \\
\sum_{i} x_{i}=1, \\
0 \leqslant x_{1} \leqslant x_{2} \leqslant \ldots \leqslant x_{n} .
\end{gathered}
$$


For any fixed $z$, our results for Problem II in section 2 show (constructively) that an associated $\mathbf{x}$ exists if and only if

$$
\begin{gathered}
a_{i}-z / w_{i} \leqslant a_{j}+z / w_{j} \quad \text { for } i \leqslant j, \\
\Sigma_{i} \max \left\{0, \max _{j \leqslant i}\left(a_{j}-z / w_{j}\right)\right\} \leqslant 1, \\
\Sigma_{i} \min _{j \geqslant i}\left(a_{j}+z / w_{j}\right) \geqslant 1 .
\end{gathered}
$$

As before, we can write

$$
z_{\min }=\max \left\{z^{\circ}, z^{*}, z^{* *}\right\}
$$

where now

$$
\begin{gathered}
z^{\circ}=\text { smallest value of } z \text { satisfying }(3.2 .30), \\
z^{*}=\text { least nonnegative } z \text { satisfying }(3.2 .31) \\
z^{* *}=\text { least nonnegative } z \text { satisfying }(3.2 .32)
\end{gathered}
$$

By a simple evaluation,

$$
z^{\circ}=\max _{i \leqslant j}\left\{\left(a_{i}-a_{j}\right) /\left[1 / w_{i}+1 / w_{j}\right]\right\} .
$$

The determination of $z^{*}$ is an instance of Problem $\mathrm{V}$ in section 2, for which we lack a solution method (unless a and $\mathbf{w}$ are similarly ordered). The determination of $z^{* *}$, in turn is an instance of section 2's Problem VI, for which a solution method is also lacking (unless a and $\mathbf{w}$ are oppositely ordered).

For the unweighted case (all $w_{j}=1$ ), as noted in section 2, Problem $\mathrm{V}$ reduces to the solvable Problem III, so that $z^{*}$ can be found; similarly finding $z^{* *}$ is an instance of the solvable "oppositely ordered" special case of Problem VI. Thus, as found in [4], the "componentwise ranking" version of the minimax adjustment problem is solvable in the unweighted case.

\subsection{Both Componentwise Bounds and Ranking}

In this subsection we consider the situation in which the "incomplete information" involves both componentwise bounds and a componentwise ranking on the probability $n$-vector in question. Thus our constraint set here is given by

$$
P=\left\{\mathbf{x}: \mathbf{L} \leqslant \mathbf{x} \leqslant \mathbf{U}, x_{1} \leqslant x_{2} \leqslant \ldots \leqslant x_{n}, \Sigma_{i} x_{i}=1\right\} .
$$

It will be convenient to define $n$-vectors $\mathbf{L}^{\prime}$ and $\mathbf{U}^{\prime}$ by

$$
L_{i}{ }^{\prime}=\max _{j \leqslant i} L_{j}, U_{i}{ }^{\prime}=\min _{j \geqslant i} U_{j} .
$$

Then it is easy to see that

$$
P=\left\{\mathbf{x}: \mathbf{L}^{\prime} \leqslant \mathbf{x} \leqslant \mathbf{U}^{\prime}, x_{1} \leqslant x_{2} \leqslant \ldots \leqslant x_{n}, \Sigma_{i} x_{i}=1\right\} .
$$

Replacing $L_{i}{ }^{\prime}$ by $\max \left\{0, L_{i}{ }^{\prime}\right\}$ and $U_{i}{ }^{\prime}$ by $\min \left\{U_{i}{ }^{\prime}, 1\right\}$ if necessary, we may assume that $\mathbf{0} \leqslant \mathbf{L}^{\prime}$ and $\mathbf{U}^{\prime} \leqslant \mathbf{l}$.

The analysis of Problem II in section 2 shows that $P$ is nonempty if and only if

$$
\begin{gathered}
\mathbf{L}^{\prime} \leqslant \mathbf{U}^{\prime}, \\
\Sigma_{i} L_{i}{ }^{\prime} \leqslant 1 \leqslant \Sigma_{i} U_{i}{ }^{\prime} .
\end{gathered}
$$

These two conditions will be assumed to hold from now on. 
For the above polyhedron $P$, and a fixed $i \epsilon\{1,2, \ldots, n\}$, we proceed as in [3] to seek the quantities $M_{i}, m_{i}$ of (1.4) and (1.5). Consider the conditions under which

$$
\mathbf{x}=\left(y_{1}, \ldots, y_{i-1}, x_{i}, y_{i}, \ldots, y_{n-1}\right) \epsilon P .
$$

These conditions are

$$
\begin{array}{cc}
L_{i}{ }^{\prime} \leqslant x_{i} \leqslant U_{i}{ }^{\prime}, & \\
L_{j}{ }^{\prime} \leqslant y_{j} \leqslant \min \left\{U_{j}{ }^{\prime}, x_{i}\right\} & \text { for } j<i, \\
\max \left\{L_{j+1}{ }^{\prime}, x_{i}\right\} \leqslant y_{j} \leqslant U_{j+1}{ }^{\prime} & \text { for } i \leqslant j<n, \\
\sum_{j} y_{j}=1-x_{i} . &
\end{array}
$$

The discussion of Problem II in section 2 shows that an $(n-1)$-vector $\mathbf{y}$ satisfying (3.3.6) through (3.3.8) exists if and only if

$$
\begin{gathered}
L_{j}{ }^{\prime} \leqslant \min \left\{U_{j}{ }^{\prime}, x_{i}\right\} \quad \text { for } j<i, \\
\max \left\{L_{j+1}{ }^{\prime}, x_{i}\right\} \leqslant U_{j+1}{ }^{\prime} \quad \text { for } i \leqslant j<n, \\
\sum_{j<i} L_{j}{ }^{\prime}+\sum_{j=i}^{n-1} \max \left\{L_{j+1}{ }^{\prime}, x_{i}\right\} \leqslant 1-x_{i}, \\
\sum_{j<i} \min \left\{U_{j}{ }^{\prime}, x_{i}\right\}+\sum_{j=i}^{n-1} U_{j+1}{ }^{\prime} \geqslant 1-x_{i} .
\end{gathered}
$$

Here (3.3.9) and (3.3.10) are automatically satisfied by virtue of (3.3.5). We can rewrite (3.3.11) and (3.3.12), respectively, as

$$
\begin{aligned}
& x_{i}+\sum_{j=i}^{n-1} \max \left\{L_{j+1}{ }^{\prime}, x_{i}\right\} \leqslant 1-\Sigma_{j<i} L_{j}{ }^{\prime}, \\
& x_{i}+\Sigma_{j<i} \min \left\{U_{j}{ }^{\prime}, x_{i}\right\} \geqslant 1-\sum_{j=i}^{n-1} U_{j+1}{ }^{\prime} .
\end{aligned}
$$

The left-hand sides of (3.3.13) and (3.3.14) are continuous increasing functions of $x_{i}$, bounded neither above nor below. They are therefore equal to their right-hand sides for unique respective values $x_{i}{ }^{+}$and $x_{i}{ }^{-}$. It follows that (3.3.13) and (3.3.14) are equivalent to $x_{i}{ }^{-} \leqslant x_{i} \leqslant x_{i}{ }^{+}$. By combination with (3.3.5), this yields

$$
M_{i}=\min \left\{U_{i}{ }^{\prime}, x_{i}{ }^{+}\right\}, m_{i}=\max \left\{L_{i}{ }^{\prime}, x_{i}{ }^{-}\right\} .
$$

To determine $M_{i}$ and $m_{i}$, it therefore suffices ${ }^{6}$ to determine $x_{i}^{+}$and $x_{i}{ }^{-}$. For this purpose, rewrite (3.3.14) as

$$
x_{i}-\Sigma_{j<i} \max \left\{0, U_{j}^{\prime}-x_{i}\right\} \geqslant 1-\Sigma_{j} U_{j}{ }^{\prime} ;
$$

thus finding $x_{i}{ }^{-}$is an instance of Problem IV in section 2, and hence can be regarded as a solved problem.

Next, rewrite (3.3.13) as

$$
x_{i}+\Sigma_{j \geqslant i} \max \left\{0, x_{i}-L_{j}{ }^{\prime}\right\} \leqslant 1-\Sigma_{j} L_{j}{ }^{\prime}=S .
$$

Clearly any solution $x_{i}$ has $x_{i} \leqslant S$, so that $z=S-x_{i} \geqslant 0$; finding the maximum value $x_{i}{ }^{+}$of the solutions $x_{i}$ of (3.3.16) is therefore equivalent to finding the minimum $z_{i} \geqslant 0$ such that

$$
z_{i}-\Sigma_{j \geqslant i} \max \left\{0,\left(S-L_{j}{ }^{\prime}\right)-z_{i}\right\} \geqslant 0,
$$

another instance of Problem IV. Thus the determination of $M_{i}$ and $m_{i}$ can be regarded as a solved problem. 
We turn now to the (weighted) minimax error selection problem, which by (1.6)-(1.8) is that of choosing number $z$ and $n$-vector $\mathbf{x}$ to minimize $z$ subject to

$$
\begin{aligned}
\max \left\{L_{i}{ }^{\prime}, M_{i}-z / w_{i}\right\} \leqslant & \left.x_{i} \leqslant \min \left\{U_{i}{ }^{\prime}, m_{i}+z / w_{i}\right\}, \quad \text { (all } i\right) \\
& \Sigma_{i} x_{i}=1, \\
x_{1} \leqslant & x_{2} \leqslant \ldots \leqslant x_{n} .
\end{aligned}
$$

For any fixed $z$, it follows from the analysis of Problem II that an $\mathbf{x}$ satisfying these three conditions exists if and only if

$$
\begin{gathered}
\left.\max _{j \leqslant i} \max \left\{L_{j}{ }^{\prime}, M_{j}-z / w_{j}\right\} \leqslant \min _{j \geqslant i} \min \left\{U_{j}{ }^{\prime}, m_{j}+z / w_{j}\right\} \quad \text { (all } i\right), \\
\Sigma_{i} \max _{j \leqslant i} \max \left\{L_{j}{ }^{\prime}, M_{j}-z / w_{j}\right\} \leqslant 1, \\
\sum_{i} \min _{j \geqslant i} \min \left\{U_{j}{ }^{\prime}, m_{j}+z / w_{j}\right\} \geqslant 1 .
\end{gathered}
$$

The constraint $z \geqslant 0$ can be imposed if desired.

Following our customary pattern, set

$$
\begin{gathered}
z^{\circ}=\text { least } z \text { satisfying (3.3.18), } \\
z^{*}=\text { least } z \geqslant 0 \text { satisfying (3.3.19), } \\
z^{* *}=\text { least } z \geqslant 0 \text { satisfying (3.3.20); }
\end{gathered}
$$

then we have for the desired minimum value of $z$,

$$
z_{\min }=\max \left\{z^{\circ}, z^{*}, z^{* *}\right\} .
$$

Since (3.3.18) can be rewritten

$$
\max \left\{L_{i}{ }^{\prime}, \max _{j \leqslant i}\left(M_{j}-z / w_{j}\right)\right\} \leqslant \min \left\{U_{i}{ }^{\prime}, \min _{j \geq i}\left(m_{j}+z / w_{j}\right)\right\},
$$

it is readily found that

$$
z^{\circ}=\max _{i \leqslant j}\left\{w_{j}\left(L_{i}{ }^{\prime}-m_{j}\right), w_{i}\left(M_{i}-U_{j}{ }^{\prime}\right),\left(M_{i}-m_{j}\right) /\left(1 / w_{i}+1 / w_{j}\right)\right\} .
$$

Since $L_{i}{ }^{\prime} \leqslant m_{i}$, while $i \leqslant j$ implies $L_{i}{ }^{\prime} \leqslant L_{j}{ }^{\prime} \leqslant m_{j}$, we have

$$
\left(w_{i}+w_{j}\right) L_{i}{ }^{\prime} \leqslant w_{i} M_{i}+w_{j} m_{j},
$$

or equivalently

$$
\left(w_{i}+w_{j}\right)\left(L_{i}{ }^{\prime}-m_{j}\right) \leqslant w_{i}\left(M_{i}-m_{j}\right)
$$

implying

$$
w_{j}\left(L_{i}{ }^{\prime}-m_{j}\right) \leqslant\left(M_{i}-m_{j}\right) /\left(1 / w_{i}+1 / w_{j}\right) .
$$

Similarly, since $m_{j} \leqslant U_{j}{ }^{\prime}$ while $i \leqslant j$ implies $M_{i} \leqslant U_{i}{ }^{\prime} \leqslant U_{j}{ }^{\prime}$, we have

$$
w_{i}\left(M_{i}-U_{j}{ }^{\prime}\right) \leqslant 0 \leqslant w_{j}\left(U_{j}{ }^{\prime}-m_{j}\right),
$$

or equivalently

$$
\left(w_{i}+w_{j}\right)\left(M_{i}-U_{j}{ }^{\prime}\right) \leqslant w_{j}\left(M_{i}-m_{j}\right),
$$

implying

$$
w_{i}\left(M_{i}-U_{j}^{\prime}\right) \leqslant\left(M_{i}-m_{j}\right) /\left(1 / w_{i}+1 / w_{j}\right)
$$


Thus (3.3.21) becomes

$$
z^{\circ}=\max _{i \leqslant j}\left\{\left(M_{i}-m_{j}\right) /\left(1 / w_{i}+1 / w_{j}\right)\right\}
$$

Next, (3.3.19) and (3.3.20) can respectively be rewritten

$$
\begin{aligned}
& \Sigma_{i} \max \left\{L_{i}{ }^{\prime}, \max _{j \leqslant i}\left(M_{j}-z / w_{j}\right)\right\} \leqslant 1, \\
& \Sigma_{i} \min \left\{U_{i}{ }^{\prime}, \min _{j \geqslant i}\left(m_{j}+z / w_{j}\right)\right\} \geqslant 1 .
\end{aligned}
$$

From (3.3.23), the determination of $z^{*}$ is clearly an instance of Problem VII in section 2. Rewriting (3.3.24) as

$$
\Sigma_{i} \max \left\{-U_{i}{ }^{\prime}, \max _{j \geq i}\left[\left(-m_{j}\right)-z / w_{j}\right]\right\} \leqslant-1
$$

shows that determining $z^{* *}$ is again an instance of Problem VII.

Thus here the bottleneck is the lack of a satisfactory solution method for Problem VII. The unweighted case (all $w_{j}=1$ ) is solvable, as in [3], since then Problem VII reduces to the solvable Problem III.

We turn now to the minimax adjustment problem, which here involves selecting a number $z$ and an $n$-vector $\mathbf{x}$ to minimize $z$, subject to

$$
\begin{gathered}
\max \left\{L_{i}{ }^{\prime}, a_{i}-z / w_{i}\right\} \leqslant x_{i} \leqslant \min \left\{U_{i}{ }^{\prime}, a_{i}+z / w_{i}\right\} \quad(\text { all } i), \\
\sum_{i} x_{i}=1, \\
x_{1} \leqslant x_{2} \leqslant \ldots \leqslant x_{n} .
\end{gathered}
$$

For any given $z$, an $\mathbf{x}$ obeying these three conditions exists if and only if

$$
\begin{gathered}
\left.\max _{j \leqslant i} \max \left\{L_{j}{ }^{\prime}, a_{j}-z / w_{j}\right\} \leqslant \min _{j \geqslant i} \min \left\{U_{j}{ }^{\prime}, a_{j}+z / w_{j}\right\} \quad \text { (all } i\right), \\
\Sigma_{i} \max _{j \leqslant i} \max \left\{L_{j}{ }^{\prime}, a_{j}-z / w_{j}\right\} \leqslant 1, \\
\Sigma_{i} \min _{j \geqslant i} \min \left\{U_{j}{ }^{\prime}, a_{j}+z / w_{j}\right\} \geqslant 1,
\end{gathered}
$$

requirements which are respectively equivalent to

$$
\begin{gathered}
\max \left\{L_{i}{ }^{\prime}, \max _{j \leqslant i}\left(a_{j}-z / w_{j}\right)\right\} \leqslant \min \left\{U_{i}{ }^{\prime}, \min _{j \geqslant i}\left(a_{j}+z / w_{j}\right)\right\}(\text { all } i), \\
\Sigma_{i} \max \left\{L_{i}{ }^{\prime}, \max _{j \leqslant i}\left(a_{j}-z / w_{j}\right)\right\} \leqslant 1, \\
\Sigma_{i} \min \left\{U_{i}{ }^{\prime}, \min _{j \geqslant i}\left(a_{j}+z / w_{j}\right)\right\} \geqslant 1 .
\end{gathered}
$$

Defining $z^{\circ}, z^{*}$ and $z^{* *}$ in analogy with the cases treated previously, we will again have

$$
z_{\min }=\max \left\{z^{\circ}, z^{*}, z^{* *}\right\}
$$

It is readily verified that

$$
z^{\circ}=\max _{i \leq j}\left\{w_{j}\left(L_{i}^{\prime}-a_{j}\right), w_{i}\left(a_{i}-U_{j}^{\prime}\right),\left(a_{i}-a_{j}\right) /\left(1 / w_{i}+1 / w_{j}\right)\right\} .
$$

If a satisfies the modified componentwise bounds, i.e. $\mathbf{L}^{\prime} \leqslant \mathbf{a} \leqslant \mathbf{U}^{\prime}$, then it follows that for $i \leqslant j$,

$$
L_{i}{ }^{\prime} \leqslant a_{i} \text { and } L_{i}{ }^{\prime} \leqslant L_{j}{ }^{\prime} \leqslant a_{j},
$$

implying

$$
w_{j}\left(L_{i}{ }^{\prime}-a_{j}\right) \leqslant 0 \leqslant w_{i}\left(a_{i}-L_{i}{ }^{\prime}\right)
$$


and thus

$$
w_{j}\left(L_{i}^{\prime}-a_{j}\right) \leqslant\left(a_{i}-a_{j}\right) /\left(1 / w_{i}+1 / w_{j}\right)
$$

similarly

$$
w_{i}\left(a_{i}-U_{j}^{\prime}\right) \leqslant w_{j}\left(U_{j}^{\prime}-a_{j}\right),
$$

leading to

$$
w_{i}\left(a_{i}-U_{j}^{\prime}\right) \leqslant\left(a_{i}-a_{j}\right) /\left(1 / w_{i}+1 / w_{j}\right) .
$$

Thus $\mathbf{L}^{\prime} \leqslant \mathbf{a} \leqslant \mathbf{U}^{\prime}$ implies that (3.3.29) simplifies to

$$
z^{\circ}=\max _{i \leqslant j}\left\{\left(a_{i}-a_{j}\right) /\left(1 / w_{i}+1 / w_{j}\right)\right\} .
$$

The form of (3.3.27) shows that finding $z^{*}$ is an instance of Problem VII, for which we lack a solution method unless a and $\mathbf{w}$ are similarly ordered. Rewriting (3.3.28) as

$$
\Sigma_{i} \max \left\{-U_{i}{ }^{\prime}, \max _{j \geq i}\left[\left(-a_{j}\right)-z / w_{j}\right]\right\} \leqslant-1
$$

shows that the determination of $z^{* *}$ is also an instance of Problem VII, for which we lack a solution method unless a and $\mathbf{w}$ are oppositely ordered. The unweighted case (all $w_{j}=1$ ) is however exhibited as solvable, in accordance with [4].

\section{Minimax Disaggregation of an Exact Distribution}

\subsection{Minimax-Error Selection}

In this section and the next, we deal with a fixed partition $\left\{S_{j}\right\}_{1}{ }^{m}$ of $\{1,2, \ldots, n\}$ into $m$ sets $S_{j}$ with at least two members each (i.e., $\left|S_{j}\right| \geqslant 2$ ). The notation

$$
\mathbf{x}(T)=\Sigma\left\{x_{i}: i \epsilon T\right\}
$$

will be employed; here $\mathbf{x}$ is an $n$-vector with components $\left\{x_{i}\right\}_{1}{ }^{n}$, and $T$ is a subset of $\{1,2, \ldots, n\}$.

The intended interpretation is that $\{1,2, \ldots, n\}$ indexes the outcomes of some chance event as classified in a relatively "fine" manner, whereas $\{1,2, \ldots, m\}$ indexes the outcomes as classified more grossly. More specifically, the "micro-outcomes" indexed by the members $i \epsilon S_{j}$ constitute in aggregate the $j$ th "macro-outcome."

In the present section, we treat several versions of the following problem: Given an initial positive probability $m$-vector $\mathbf{s}$, with component $s_{j}$ representing the probability of the $j$ th macro-outcome, determine in a systematic way a probability $n$-vector $\mathbf{x}$, whose component $x_{i}$ is to be regarded as the probability of the $i$ th micro-outcome.

Suppose first that no further conditions are placed on $\mathbf{x}$. Then the appropriate constraint set for $\mathbf{x}$ is the polyhedron

$$
P=\left\{\mathbf{x}: \mathbf{x} \geqslant 0, \mathbf{x}\left(S_{j}\right)=s_{j} \text { for } 1 \leqslant j \leqslant m\right\} .
$$

Our approach to finding a suitable $\mathbf{x}$ is to solve the weighted minimax selection problem for $P$, i.e., to determine $\mathbf{x} \epsilon P$ so as to minimize

$$
F(\mathbf{x} ; \mathbf{w})=\max _{\mathbf{y} \epsilon} P \max _{i} w_{i}\left|x_{i}-y_{i}\right| .
$$

Let $\mathbf{x}_{j}$ and $\mathbf{y}_{j}$ denote variable $\left|S_{j}\right|$ vectors whose components are indexed by the members $i \epsilon S_{j}$, and in $\mathbf{x}_{j}$-space define the polyhedron

$$
P_{j}=\left\{\mathbf{x}_{j}: \mathbf{x}_{j} \geqslant 0, \mathbf{x}_{j}\left(S_{j}\right)=s_{j}\right\} .
$$


Then clearly $P$ is the Cartesian product of the $P_{j}$ 's; if we put

$$
F_{j}\left(\mathbf{x}_{j} ; \mathbf{w}_{j}\right)=\max \left\{w_{i}\left|x_{i}-y_{i}\right|: i \epsilon S_{j} ; \mathbf{y}_{j} \epsilon P_{j}\right\}
$$

then it follows that

$$
F(\mathbf{x} ; \mathbf{w})=\max _{j} F_{j}\left(\mathbf{x}_{j} ; \mathbf{w}_{j}\right) .
$$

The consequence is that the weighted minimax error selection problem for $P$ decomposes into $m$ independent subproblems, the $j$ th of which is the weighted minimax error selection problem for $P_{j}$.

To solve the $j$ th subproblem, it is convenient to observe that the $M_{i}=M_{i}(P)$ and $m_{i}=m_{i}(P)$ of (1.4) and (1.5) are given, for $i \epsilon S_{j}$, by

$$
M_{i}=\max \left\{x_{i}: \mathbf{x}_{j} \epsilon P_{j}\right\}
$$

The analog of (1.3), namely

$$
F_{j}\left(\mathbf{x}_{j} ; \mathbf{w}_{j}\right)=\max _{i \epsilon S_{j}} \max \left\{w_{i}\left(M_{i}-x_{i}\right), w_{i}\left(x_{i}-m_{i}\right)\right\},
$$

is readily seen to hold. Thus the $j$ th subproblem can be reduced to selecting a number $z_{j}$ and an $\left|S_{j}\right|$ vector $\mathbf{x}_{j}$ to minimize $z_{j}$ subject to

$$
\begin{aligned}
& \max \left\{0, M_{i}-z_{j} / w_{i}\right\} \leqslant x_{i} \leqslant m_{i}+z_{j} / w_{i} \quad\left(\text { all } i \epsilon S_{j}\right), \\
& \mathbf{x}\left(S_{j}\right)=s_{j}, \\
& \mathbf{x}_{j} \in P_{j} \text {. }
\end{aligned}
$$

For the particular $P$ and $P_{j}$ given by (4.1.1) and (4.1.3), the constraint (4.1.11) is redundant in the presence of (4.1.9) and (4.1.10). By the analysis of Problem I in section 2, for any given $z_{j} \geqslant 0$ an associated $\mathbf{x}_{j}$ exists if and only if

$$
\begin{gathered}
M_{i}-z_{j} / w_{i} \leqslant m_{i}+z_{j} / w_{i}, \\
\Sigma\left\{\max \left[0, M_{i}-z_{j} / w_{i}\right]: i \epsilon S_{j}\right\} \leqslant s_{j}, \\
\Sigma\left\{m_{i j}+z_{j} / w_{i}: i \epsilon S_{j}\right\} \geqslant s_{j} .
\end{gathered}
$$

With the familiar formula

$$
z_{j}^{\min }=\max \left\{z_{j}^{\circ}, z_{j}^{*}, z_{j}^{* *}\right\}
$$

we have

$$
z_{j}^{\circ}=\frac{1}{2} \max \left\{w_{i}\left(M_{i}-m_{i}\right): i \epsilon S_{j}\right\}
$$

The determination of $z_{j}^{*}$ from (4.1.13) is an instance of (solvable) Problem III in section 2. And (4.1.14) yields directly

$$
z_{j}^{* * i}=\max \left\{0,\left[s_{j}-\Sigma_{i \epsilon S_{j}} m_{i}\right] / \Sigma_{i \epsilon S_{j}}\left(1 / w_{i}\right)\right\} .
$$

Thus this problem can be regarded as solved, once the $M_{i}$ and $m_{i}$ are known. But for the present $P_{j}$, it is easy to check that

$$
M_{i}=s_{j} \text { and } m_{i}=0 \quad\left(i \epsilon S_{j}\right)
$$

so that (4.1.15) yields

$$
z^{\circ}=\max _{j} z_{j}^{\circ}=\frac{1}{2} \max _{j}\left\{s_{j} \max _{i \epsilon S_{j}}\left(w_{i}\right)\right\},
$$


while (4.1.16) yields

$$
z^{* *}=\max _{j} z_{j}^{* *}=\max _{j}\left\{s_{j} / \Sigma_{i \epsilon S_{j}}\left(1 / w_{i}\right)\right\}
$$

Using (4.1.8), we have

$$
z_{\min }=\max \left\{z^{\circ}, z^{* *}, \max _{j} z_{j}^{*}\right\}
$$

with $z^{\circ}$ and $z^{* *}$ given by (4.1.17) and (4.1.18), and $z_{j}^{*}$ the solution of Problem III applied to

$$
\Sigma\left\{\max \left[0, s_{j}-z_{j} / w_{i}\right]: i \epsilon S_{j}\right\} \leqslant s_{j} .
$$

It is not hard to show, however, that $z_{j}^{* *} \leqslant z_{j}{ }^{\circ}$, so that $z^{* *} \leqslant z^{\circ}$ and (4.1.19) becomes

$$
z_{\min }=\max \left\{z^{\circ}, \max _{j} z_{j}^{*}\right\}=\max _{j}\left\{z_{j}^{\circ}, z_{j}^{*}\right\} .
$$

To consider the order relation between $z_{j}{ }^{\circ}$ and $z_{j}{ }^{*}$, note that $z_{j}{ }^{*} \geqslant z_{j}{ }^{\circ}$ holds if and only if the opposite of (4.1.20) is satisfied for $z_{j}=z_{j}^{\circ}$, i.e., if and only if

$$
\Sigma\left\{\max \left[0,1-\left(\max _{k \epsilon S_{j}} w_{k}\right) / 2 w_{i}\right]: i \epsilon S_{j}\right\} \geqslant 1 .
$$

This will in particular be true, in the semi-weighted case when all $w_{i}\left(i \epsilon S_{j}\right)$ have the same value $W_{j}$, so that for each such $j$

$$
z_{j}^{\min }=z_{j}^{*}=W_{j} s_{j}\left[1-1 /\left|S_{j}\right|\right] .
$$

Should this apply for all $j \epsilon\{1,2, \ldots, m\}$, we have

$$
z_{\min }=\max _{j} W_{j} s_{j}\left[1-1 /\left|S_{j}\right|\right],
$$

which in particular holds for the unweighted version of the original problem.

We next treat the solvability of the weighted minimax selection problem under further restrictions on $\mathbf{x}$. To preserve the decomposition which guided the solution in the previous case, these constraints must maintain the independence of the $m$ subproblems, i.e., must deal individually with the subvectors $\mathbf{x}_{j}$ of $\mathbf{x}$.

Consider first the case of componentwise bounds on $\mathbf{x}$, so that (4.1.1) is replaced by

$$
P=\left\{\mathbf{x}: 0 \leqslant \mathbf{L} \leqslant \mathbf{x} \leqslant \mathbf{U}, \mathbf{x}\left(S_{j}\right)=s_{j} \text { for } 1 \leqslant j \leqslant m\right\},
$$

where $0 \leqslant \mathbf{L} \leqslant \mathbf{U}$ may be assumed at the outset. Let $\mathbf{L}_{j}$ and $\mathbf{U}_{j}$ be the subvectors of $\mathbf{L}$ and $\mathbf{U}$ associated with $S_{j}$. Then the $j$ th subproblem has constraint set

$$
P_{j}=\left\{\mathbf{x}_{j}: \mathbf{x}_{j}\left(S_{j}\right)=s_{j}, \mathbf{L}_{j} \leqslant \mathbf{x}_{j} \leqslant \mathbf{U}_{j}\right\} .
$$

The weighted minimax selection problem for $P_{j}$ can be solved as in subsection 3.1 of this paper; indeed, a rescaling by $1 / s_{j}$ reduces it to the first problem treated in that subsection. The feasibility conditions, in addition to $\mathbf{L}_{j} \leqslant \mathbf{U}_{j}$, are given by

$$
\mathbf{L}\left(S_{j}\right)=\Sigma\left\{L_{i}: i \epsilon S_{j}\right\} \leqslant s_{j} \leqslant \Sigma\left\{U_{i}: i \epsilon S_{j}\right\}=\mathbf{U}\left(S_{j}\right)
$$

for the $j$ th subproblem.

Second, a componentwise ranking might be imposed on each of the subvectors $\mathbf{x}_{j}$. The $j$ th subproblem, after rescaling by $1 / s_{j}$, is then of the type treated in subsection 3.2 ; in particular we have a solution method in the semiweighted case $\left(w_{i}=W_{j}\right.$ for all $\left.i \epsilon S_{j}\right)$ - or more generally, if the ordering of the subvector $\mathbf{w}_{j}$ of $\mathbf{w}$ is consistent with the ranking imposed on $\mathbf{x}_{j}$.

Third, both componentwise bounds and a componentwise ranking might be imposed on each $\mathbf{x}_{j}$. 
Rescaling then yields the type of problem taken up in subsection 3.3; again we have a solution method if $w_{i}=W_{j}$ for all $i \epsilon S_{j}$.

We can of course have "mixed" cases of the last three situations: some $\mathbf{x}_{j}$ may be subjected to componentwise bounds only, others to a ranking only, and still others to both. So long as the subproblems remain independent, one simply applies to each subproblem that part of the preceding discussion which is applicable.

\subsection{Minimax Adjustment}

Here the polyhedra $P$ and $P_{j}$ are as in the preceding subsection, but the function to be minimized is

$$
G(\mathbf{x} ; \mathbf{w})=\max _{j} G_{j}\left(\mathbf{x}_{j} ; \mathbf{w}_{j}\right),
$$

where

$$
G_{j}\left(\mathbf{x}_{j} ; \mathbf{w}_{j}\right)=\max \left\{w_{i}\left|x_{i}-a_{i}\right|: i \epsilon S_{j}\right\}
$$

and $\mathbf{a}$ is a given probability $n$-vector. Again we have $m$ independent subproblems; the $j$ th of them is to select number $z_{j}$ and $\left|S_{j}\right|$-vector $\mathbf{x}_{j}$ so as to minimize $z_{j}$ subject to

$$
\begin{gathered}
\max \left\{0, a_{i}-z_{j} / w_{i}\right\} \leqslant x_{i} \leqslant a_{i}+z_{j} / w_{i} \quad\left(\text { all } i \epsilon S_{j}\right), \\
\mathbf{x}\left(S_{j}\right)=s_{j}, \\
\mathbf{x}_{j} \in P_{j} .
\end{gathered}
$$

The first case considered is that in which $\mathbf{x}$ is constrained only by the aggregation condition (4.2.4), so that (4.2.5) is redundant. For any $z_{j} \geqslant 0$, there exists an $\mathbf{x}_{j}$ satisfying (4.2.3) and (4.2.4) if and only if

$$
\begin{gathered}
a_{i}-z_{j} / w_{i} \leqslant a_{i}+z_{j} / w_{i} \quad\left(\text { all } i \epsilon S_{j}\right), \\
\Sigma\left\{\max \left[0, a_{i}-z_{j} / w_{i}\right]: i \epsilon S_{j}\right\} \leqslant s_{j}, \\
\Sigma\left\{a_{i}+z_{j} / w_{i}: i \epsilon S_{j}\right\} \geqslant s_{j} .
\end{gathered}
$$

Here (4.2.6) is automatically satisfied, so that with the familiar notation,

$$
z_{j}^{\min }=\max \left\{z_{j}^{*}, z_{j}^{* *}\right\} .
$$

From (4.2.8) one obtains directly

$$
z_{j}^{* *}=\max \left\{0, s_{j}-\mathbf{a}\left(S_{j}\right)\right\} / \Sigma\left\{1 / w_{i}: i \epsilon S_{j}\right\} .
$$

The determination of $z_{j}^{*}$, as the minimum value of $z_{j}$ satisfying both $z_{j} \geqslant 0$ and (4.2.7), is an instance of Problem III in section 2, and so can be regarded as solved. Note that if $s_{j} \geqslant \mathbf{a}\left(S_{j}\right)$, then (4.2.7) is satisfied by $z_{j}=0$, so that (4.2.9) yields $z_{j}^{\min }=z_{j}^{* *}$; on the other hand, if $s_{j} \leqslant \mathbf{a}\left(S_{j}\right)$, then $z_{j}^{\min }=z_{j}^{*}$.

The remaining cases are those in which $\mathbf{x}_{j}$ may be constrained by componentwise bounds, a componentwise ranking or both. Their treatment resembles that in the previous subsection, drawing on the analyses of minimax adjustment problems in subsections 3.1, 3.2 and 3.3. The case of componentwise bounds is solvable in general, but at present the other two cases admit satisfactory solution only when $w_{i}=W_{j}$ for all $i \epsilon S_{j}$.

\section{Minimax Disaggregation of a "Fuzzy" Distribution}

\subsection{No Other Information}

In section 4, it was assumed that a probability vector s describing the probabilities of the chance event's "macro-outcomes" is known exactly. This assumption played an essential technical 
role in simplifying the analysis: the aggregation conditions $\mathbf{x}\left(S_{j}\right)=s_{j}$, together with the fact that $\Sigma_{j} s_{j}$ $=1$, implied that

$$
\Sigma_{i} x_{i}=1
$$

Thus it was not necessary to impose (5.0.1) as an explicit constraint; this condition, relating the $m$ subvectors $\left\{\mathbf{x}_{j}\right\}_{1}{ }^{m}$, would have ruined section 4's approach of dealing with $m$ independent subproblems each involving a single $\mathbf{x}_{j}$.

For some situations, however, the assumption of an exactly known initial distribution s may well be unacceptable. In the present section, we replace the "exact" aggregation conditions $\mathbf{x}\left(S_{j}\right)=s_{j}$ by "approximate aggregation" conditions

$$
s_{j}{ }^{-} \leqslant \mathbf{x}\left(S_{j}\right) \leqslant s_{j}{ }^{+} \quad(1 \leqslant j \leqslant m),
$$

involving the components of given $m$-vectors $\mathbf{s}^{-}$and $\mathbf{s}^{+}$which (cf. Problem I in sec. 2) we may assume to satisfy

$$
\begin{gathered}
\mathbf{0} \leqslant \mathbf{s}^{-} \leqslant \mathbf{s}^{+} \leqslant \mathbf{1}, \\
\Sigma_{j} s_{j}^{-} \leqslant 1 \leqslant \Sigma_{j} s_{j}{ }^{+} .
\end{gathered}
$$

This loosening means that the constraint (5.0.1) must be explicitly imposed; the $m$ subproblems are no longer independent, but their interdependence is limited to the single condition (5.0.1).

The simplest weighted minimax-error selection problem of this type has

$$
P=\left\{\mathbf{x}: \mathbf{x} \geqslant 0, \Sigma_{i} x_{i}=1, \quad s_{j}^{-} \leqslant \mathbf{x}\left(S_{j}\right) \leqslant s_{j}{ }^{+} \text {for } 1 \leqslant j \leqslant m\right\} .
$$

The first step in the analysis is to determine the $M_{i}$ and $m_{i}$ for this polyhedron $P$.

For a fixed $J \epsilon\{1,2, \ldots, m\}$, a fixed $i \epsilon S_{J}$, and a fixed $x_{i} \epsilon[0,1]$, consider Problem VIII in section 2 with $S=1$ and

$$
\begin{gathered}
A_{i}=B_{i}=x_{i}, \\
A_{k}=0 \text { and } B_{k}=1 \text { for } k \epsilon\{1,2, \ldots, n\}-\{i\} .
\end{gathered}
$$

Of the conditions for a solution to exist, (2.30) is satisfied automatically. Condition (2.31) is satisfied for $j \neq J$, while $j=J$ yields

$$
\max \left\{s_{J^{-}}, x_{i}\right\} \leqslant \min \left\{s_{J}^{+}, x_{i}+\left|S_{J}\right|-1\right\}
$$

Finally, (2.32) yields

$$
\begin{gathered}
\sum_{j \neq J} s_{j}^{-}+\max \left\{s_{J}^{-}, x_{i}\right\} \leqslant 1, \\
\sum_{j \neq J} s_{j}^{+}+\min \left\{s_{J}^{+}, x_{i}+\left|S_{J}\right|-1\right\} \geqslant 1 .
\end{gathered}
$$

$M_{i}$, as the largest value of $x_{i}$ satisfying the last three conditions, is given by

$$
M_{i}=M_{J}^{*}=\min \left\{s_{J}^{+}, 1-\Sigma_{j \neq J} s_{j}^{-}\right\} \quad\left(i \epsilon S_{J}\right) ;
$$

similarly we have

$$
m_{i}=0 .
$$

Next, (1.6)-(1.8) yield

$$
\begin{gathered}
\left.\max \left\{0, M_{i}-z / w_{i}\right\} \leqslant x_{i} \leqslant z / w_{i} \quad \text { (all } i\right), \\
\sum_{i} x_{i}=1, \\
s_{j}{ }^{-} \leqslant \mathbf{x}\left(S_{j}\right) \leqslant s_{j}{ }^{+} \quad(\text { all } j) .
\end{gathered}
$$


By the analysis of Problem VIII in section 2, for any $z \geqslant 0$ an associated $\mathbf{x}$ exists if and only if

$$
\begin{gathered}
M_{i}-z / w_{i} \leqslant z / w_{i} \quad(\text { all } i), \\
\max \left\{s_{j}^{-}, \Sigma_{i \epsilon S_{j}} \max \left\{0, M_{i}-z / w_{i}\right\}\right\} \leqslant \min \left\{s_{j}^{+}, z \Sigma_{i \epsilon S_{j}}\left(1 / w_{i}\right)\right\}, \\
\Sigma_{j} \max \left\{s_{j}^{-}, \Sigma_{i \epsilon S_{j}} \max \left\{0, M_{i}-z / w_{i}\right\}\right\} \leqslant 1, \\
\Sigma_{j} \min \left\{s_{j}^{+}, z \Sigma_{i \epsilon S_{j}}\left(1 / w_{i}\right)\right\} \geqslant 1 .
\end{gathered}
$$

We wish to find the smallest $z \geqslant 0$ satisfying these four conditions.

The first condition, (5.0.10), yields

$$
\begin{aligned}
z \geqslant z^{\circ} & =\max _{j} z_{j}^{\circ} \\
& =\max _{j}\left[\frac{1}{2} M_{j}^{*} \max _{i \epsilon S_{j}} w_{i}\right] .
\end{aligned}
$$

with $M_{j}^{*}$ given by (5.0.8). The second condition, (5.0.11), is equivalent to the following trio of conditions. One is

$$
z \geqslant z^{\circ \circ}=\max _{j}\left[s_{j}-/ \Sigma_{i \epsilon S_{j}}\left(1 / w_{i}\right)\right]
$$

A second is

$$
\Sigma_{i \epsilon S_{j}} \max \left\{0, M_{j}^{*}-z / w_{i}\right\} \leqslant s_{j}^{+} \quad(\text { all } j) .
$$

If $z_{j}{ }^{\circ \circ 0}$ is the smallest $z \geqslant 0$ satisfying the $j$ th of these requirements (5.0.16), then

$$
z \geqslant z^{\circ 00}=\max _{j} z_{j}^{\circ \circ \circ} \text {. }
$$

The determination of $z_{j}{ }^{\circ 00}$ is a simple case ( $\mathbf{Z}$ has equal components) of Problem III in section 2. The third "fragment" of (5.0.11) is

$$
\Sigma_{i \epsilon S_{j}} \max \left\{0, M_{j}^{*}-z / w_{i}\right\} \leqslant z \Sigma_{i \epsilon S_{j}}\left(1 / w_{i}\right) \quad(\text { all } j),
$$

if $z_{j}{ }^{\circ 000}$ is the smallest $z \geqslant 0$ satisfying the $j$ th of these, then

$$
z \geqslant z^{0000}=\max _{j} z_{j}^{0000} \text {. }
$$

The determination of $z_{j}^{\circ \circ}$ is an instance (again $\mathbf{Z}$ has equal components) of Problem IV in section 2.

The import of the last paragraph is that we have satisfactory solution methods for finding the least $z \geqslant 0$ satisfying (5.0.10) and (5.0.11). Before going on to (5.0.12) and (5.0.13), some additional comments can be made concerning this "solved" part of the problem. First, since

$$
1 / \Sigma_{i \epsilon S_{j}}\left(1 / w_{i}\right) \leqslant \frac{1}{2} \max _{i \epsilon S_{j}} w_{i},
$$

and

$$
s_{j}{ }^{-} \leqslant s_{j}{ }^{+}, \quad s_{j}^{-} \leqslant 1-\Sigma_{J \neq j} s_{J}{ }^{-},
$$

it follows from (5.0.8), (5.0.14) and (5.0.15) that

$$
z^{\circ \circ} \leqslant z^{\circ}
$$

Second, we will show that

$$
z^{0000} \leqslant z^{\circ}
$$

by proving that

$$
z_{j}^{0000} \leqslant z_{j}^{\circ}=\frac{1}{2} M_{j}^{*} \max _{i \epsilon S_{j}} w_{i} .
$$


Since $z_{j}{ }^{000}$ is defined in terms of (5.0.18), it suffices to show that (5.0.18) is satisfied for $z=z_{j}^{\circ}$, i.e., that

$$
2 z_{j}{ }^{\circ} \sum_{i \epsilon S_{j}}\left(1 / w_{i}\right) \geqslant \sum_{i \epsilon S_{j}} \max \left\{z_{j}{ }^{\circ} w_{i}, M_{j}^{*}\right\} .
$$

This follows from a termwise comparison, the explicit expression for $z_{j}{ }^{\circ}$ being used to show that $2 z_{j}{ }^{\circ} / w_{i} \geqslant M_{j}^{*}$.

We turn now to (5.0.12) and (5.0.13). Let $z^{* *}$ be the least $z \geqslant 0$ satisfying (5.0.13), which we rewrite as

$$
\Sigma_{j} \max \left\{0, s_{j}^{+}-z \Sigma_{i \epsilon S_{j}}\left(1 / w_{i}\right)\right\} \leqslant \Sigma_{j} s_{j}^{+}-1 .
$$

Then the determination of $z^{* *}$ is an instance of Problem III in section 2, and so can be regarded as a solved problem.

Next, let $z^{*}$ be the least $z \geqslant 0$ satisfying (5.0.12), so that the minimum value of $z$ subject to (5.0.10)-(5.0.13) can be written

$$
z_{\min }=\max \left\{z^{\circ}, z^{\circ 0 \circ}, z^{* *}, z^{*}\right\},
$$

where $z^{\circ \circ}$ and $z^{\circ 000}$ are ruled out of contention by (5.0.19) and (5.0.20), $z^{\circ}$ is given explicitly by (5.0.14), and the determination of $z^{\circ 00}$ and $z^{* *}$ are solved problems. The only remaining element in the solution of this weighted minimax-error selection problem is the determination of $z^{*}$ from (5.0.12), which we rewrite as

$$
\Sigma_{j} \max \left\{s_{j}^{-}, \Sigma_{i \epsilon S_{j}} \max \left\{0 ; M_{j}^{*}-z / w_{i}\right\}\right\} \leqslant 1
$$

with $M_{j}^{*}$ given by (5.0.8).

This last element, however, is an instance of (solved) Problem IX in section 2, so that we have assembled a solution method for this weighted minimax selection problem. which

A simpler solution method is at hand for what we have called the semi-weighted case, that in

$$
w_{i}=W_{j} \quad \text { for all } i \epsilon S_{j} .
$$

In that case (5.0.23) can be written as

$$
\Sigma_{j} \max \left\{s_{j}{ }^{-},\left|S_{j}\right| \max \left\{0, M_{j}^{*}-z / W_{j}\right\}\right\} \leqslant 1,
$$

which simplifies (since $s_{j}^{-} \geqslant 0$ ) to

$$
\Sigma_{j} \max \left\{s_{j}^{-},\left|S_{j}\right|\left(M_{j}^{*}-z / W_{j}\right)\right\} \leqslant 1
$$

and thus to

$$
\Sigma_{j} \max \left\{0,\left[\left|S_{j}\right| M_{j}^{*}-s_{j}^{-}\right]-z\left|S_{j}\right| / W_{j}\right\} \leqslant 1-\Sigma_{j} s_{j}{ }^{-} .
$$

Hence determining $z^{*}$ is an instance of Problem III in section 2. Moreover, (5.0.14) and (5.0.16) yield

$$
\begin{gathered}
z_{j}^{\circ}=\frac{1}{2} M_{j}{ }^{*} W_{j}, \\
z_{j}^{\circ \circ \circ}=\max \left\{0, W_{j}\left[M_{j}^{*}-s_{j}^{+} /\left|S_{j}\right|\right]\right\},
\end{gathered}
$$

while $z^{* *}$ is the least $z \geqslant 0$ satisfying

$$
\Sigma_{j} \max \left\{0, s_{j}^{+}-z\left|S_{j}\right| / W_{j}\right\} \leqslant \Sigma_{j} s_{j}^{+}-1
$$

so that its determination is an instance of Problem III. 
We turn now to the associated minimax adjustment problem. Relations (1.10)-(1.12) yield

$$
\begin{gathered}
\left.\max \left\{0, a_{i}-z / w_{i}\right\} \leqslant x_{i} \leqslant a_{i}+z / w_{i}, \quad \text { (all } i\right) \\
\Sigma_{i} x_{i}=1, \\
s_{j}^{-} \leqslant \mathbf{x}\left(S_{j}\right) \leqslant s_{j}^{+} \quad(\text { all } j) .
\end{gathered}
$$

This instance of Problem VIII has a solution if and only if

$$
\begin{gathered}
\max \left\{0, a_{i}-z / w_{i}\right\} \leqslant a_{i}+z / w_{i} \quad(\text { all } i), \\
\max \left\{s_{j^{-}}, \Sigma_{i \epsilon S_{j}} \max \left\{0, a_{i}-z / w_{i}\right\}\right\} \leqslant \min \left\{s_{j}^{+}, \Sigma_{i \epsilon S_{j}}\left(a_{i}+z / w_{i}\right)\right\}, \\
\Sigma_{j} \max \left\{s_{j}^{-}, \Sigma_{i \epsilon S_{j}} \max \left\{0, a_{i}-z / w_{i}\right\}\right\} \leqslant 1, \\
\Sigma_{j} \min \left\{s_{j}^{+}, \Sigma_{i \epsilon S_{j}}\left(a_{i}+z / w_{i}\right)\right\} \geqslant 1 .
\end{gathered}
$$

Condition (5.0.29) is satisfied for all $z \geqslant 0$. Relation (5.0.30) is equivalent to the pair of conditions

$$
\begin{gathered}
\Sigma_{i \in S_{j}} \max \left\{0, a_{i}-z / w_{i}\right\} \leqslant s_{j}^{+}, \\
\Sigma_{i \epsilon S_{j}}\left(a_{i}+z / w_{i}\right) \geqslant s_{j}^{-} .
\end{gathered}
$$

Finding the smallest $z \geqslant 0$ to satisfy the first of these relations is an instance of Problem III; the second is equivalent to

$$
z \geqslant\left[s_{j}^{-}-\mathbf{a}\left(S_{j}\right)\right] / \Sigma_{i \epsilon S_{j}}\left(1 / w_{i}\right)
$$

Finding the least $z \geqslant 0$ obeying (5.0.31) falls under Problem IX, while the same question for (5.0.32), which can be rewritten

$$
\Sigma_{j} \max \left\{0,\left[s_{j}^{+}-\mathbf{a}\left(S_{j}\right)\right]-z \Sigma_{i \epsilon S_{j}}\left(1 / w_{i}\right)\right\} \leqslant \Sigma_{j} s_{j}^{+}-1
$$

is an instance of Problem III.

\subsection{Componentwise Bounds}

As noted earlier in this section, the problems just treated are the simplest ones in the context of disaggregating an approximate distribution. We turn now to a more complex case in which $\mathbf{x}$ is also subjected to componentwise bounds, described by $n$-vectors $\mathbf{L}$ and $\mathbf{U}$ with

$$
\mathbf{0} \leqslant \mathbf{L} \leqslant \mathbf{U} \leqslant \mathbf{1}, \quad \Sigma_{i} L_{i} \leqslant 1 \leqslant \Sigma_{i} U_{i}
$$

Thus the constraint set is given by

$$
P=\left\{\mathbf{x}: \Sigma_{i} x_{i}=1, \mathbf{L} \leqslant \mathbf{x} \leqslant \mathbf{U}, s_{j}^{-} \leqslant \mathbf{x}\left(S_{j}\right) \leqslant s_{j}{ }^{+} \text {for } 1 \leqslant j \leqslant m\right\} .
$$

To simplify later notation, we can without loss of generality adjust $\mathbf{s}^{-}$and $\mathbf{s}^{+}$so that

$$
\mathbf{L}\left(S_{j}\right) \leqslant s_{j}^{-} \leqslant s_{j}^{+} \leqslant \mathbf{U}\left(S_{j}\right)
$$

The conditions for $P$ to be nonempty are given by the analysis of Problem VIII in section 2; besides (5.1.1) and (5.1.3), they are

$$
\Sigma_{j} s_{j}^{-} \leqslant 1 \leqslant \Sigma_{j} s_{j}^{+}
$$


We begin as before by determining the appropriate $M_{i}$ and $m_{i}$. For a fixed $J \epsilon\{1,2, \ldots, m\}$, a fixed $i \epsilon S_{J}$, and a fixed $x_{i} \epsilon\left[L_{i}, U_{i}\right]$, consider Problem VIII in section 2 with $S=1, A_{i}=B_{i}=x_{i}$, and

$$
A_{k}=L_{k}, B_{k}=U_{k} \text { for all } k \neq i \text {. }
$$

Of the conditions for a solution to exist, (2.30) is automatically satisfied, as is (2.31) for all $j \neq J$. For $j=J,(2.31)$ yields

$$
\max \left\{s_{J^{-}}, \mathbf{L}\left(S_{J}\right)+x_{i}-L_{i}\right\} \leqslant \min \left\{s_{J}{ }^{+}, \mathbf{U}\left(S_{J}\right)-\left(U_{i}-x_{i}\right)\right\}
$$

while (2.32) translates into

$$
\begin{gathered}
\max \left\{s_{J^{-}}, \mathbf{L}\left(S_{J}\right)+x_{i}-L_{i}\right\}+\Sigma_{j \neq J} s_{j}^{-} \leqslant 1, \\
\min \left\{s_{j}{ }^{+}, \mathbf{U}\left(S_{J}\right)-\left(U_{i}-x_{i}\right)\right\}+\Sigma_{j \neq J} s_{j}{ }^{+} \geqslant 1 .
\end{gathered}
$$

From the last three inequalities, it follows that

$$
\begin{aligned}
& M_{i}=\min \left\{U_{i}, s_{J}{ }^{+}+L_{i}-\mathbf{L}\left(S_{J}\right), 1-\Sigma_{j \neq J s j^{-}}+L_{i}-\mathbf{L}\left(S_{J}\right)\right\}, \\
& m_{i}=\max \left\{L_{i}, s_{J}{ }^{-}+U_{i}-\mathbf{U}\left(S_{J}\right), 1-\Sigma_{j \neq J} s_{j}{ }^{+}+U_{i}-\mathbf{U}\left(S_{J}\right)\right\} .
\end{aligned}
$$

Relations (1.6)-(1.8) yield

$$
\begin{gathered}
\max \left\{L_{i}, M_{i}-z / w_{i}\right\} \leqslant x_{i} \leqslant \min \left\{U_{i}, m_{i}+z / w_{i}\right\}, \\
\sum_{i} x_{i}=1, \\
s_{j}^{-} \leqslant \mathbf{x}\left(S_{j}\right) \leqslant s_{j}^{+} .
\end{gathered}
$$

By the analysis of Problem VIII in section 2, this (for given $z$ ) has a solution $\mathbf{x}$ if and only if

$$
\begin{gathered}
\max \left\{L_{i}, M_{i}-z / w_{i}\right\} \leqslant \min \left\{U_{i}, m_{i}+z / w_{i}\right\}, \\
\max \left\{s_{j}^{-}, \Sigma_{i \epsilon S_{j}} \max \left\{L_{i}, M_{i}-z / w_{i}\right\}\right\} \leqslant \min \left\{s_{j}^{+}, \Sigma_{i \epsilon S_{j}} \min \left\{U_{i}, m_{i}+z / w_{i}\right\}\right\} . \\
\Sigma_{j} \max \left\{s_{j}^{-}, \Sigma_{i \epsilon S_{j}} \max \left\{L_{i}, M_{i}-z / w_{i}\right\}\right\} \leqslant 1, \\
\Sigma_{j} \min \left\{s_{j}{ }^{+}, \Sigma_{i \epsilon S_{j}} \min \left\{U_{i}, m_{i}+z / w_{i}\right\}\right\} \geqslant 1 .
\end{gathered}
$$

We seek the smallest value $z_{\min }$ of $z \geqslant 0$ which satisfies these four conditions.

Let $z^{\circ}$ be the least $z$ satisfying (5.1.10). As in (3.1.8), the result is

$$
z^{\circ}=\frac{1}{2} \max _{i} w_{i}\left(M_{i}-m_{i}\right),
$$

where $M_{i}-m_{i}$ can be evaluated using (5.1.8-9).

The new information in (5.1.11) is given by the pair of inequalities

$$
\begin{aligned}
& \sum_{i \epsilon S_{j}} \max \left\{L_{i}, M_{i}-z / w_{i}\right\} \leqslant s_{j}{ }^{+}, \\
& \sum_{i \epsilon S_{j}} \min \left\{U_{i}, m_{i}+z / w_{i}\right\} \geqslant s_{j}{ }^{-},
\end{aligned}
$$

which can be rewritten

$$
\begin{aligned}
& \sum_{i \epsilon S_{j}} \max \left\{0,\left(M_{i}-L_{i}\right)-z / w_{i}\right\} \leqslant s_{j}^{+}-\mathbf{L}\left(S_{j}\right), \\
& \sum_{i \epsilon S_{j}} \max \left\{0,\left(U_{i}-m_{i}\right)-z / w_{i}\right\} \leqslant \mathbf{U}\left(S_{j}\right)-s_{j}^{-}
\end{aligned}
$$

Let $z_{j}^{*}$ and $z_{j}^{* *}$ denote the smallest value of $z \geqslant 0$ satisfying (5.1.15) or (5.1.16) respectively, and put

$$
z^{*}=\max _{j} z_{j}^{*}, z^{* *}=\max _{j} z_{j}^{* *} .
$$


Then finding $z_{j}^{*}$ and $z_{j}^{* *}$ are instances of Problem III in section 2, so determining $z^{*}$ and $z^{* *}$ can be regarded as solved problems.

Now let $z^{* * *}$ be the least $z \geqslant 0$ satisfying (5.1.12), which we rewrite as

$$
\Sigma_{j} \max \left\{s_{j}^{-}-\mathbf{L}\left(S_{j}\right), \Sigma_{i \epsilon S_{j}} \max \left\{0,\left(M_{i}-L_{i}\right)-z / w_{i}\right\}\right\} \leqslant 1-\Sigma_{i} L_{i},
$$

and let $z^{* * * *}$ be the least $z \geqslant 0$ satisfying (5.1.13), which we rewrite as

$$
\Sigma_{j} \max \left\{\mathbf{U}\left(S_{j}\right)-s_{j}{ }^{+}, \Sigma_{i \epsilon S_{j}} \max \left\{0,\left(U_{i}-m_{i}\right)-z / w_{i}\right\}\right\} \leqslant \Sigma_{i} U_{i}-1
$$

Then

$$
z_{\min }=\max \left\{z^{\circ}, z^{*}, z^{* *}, z^{* * *}, z^{* * * *}\right\},
$$

where the determination of $z^{\circ}, z^{*}$ and $z^{* *}$ has already been discussed. But finding $z^{* * *}$ and $z^{* * * *}$ are instances of (solved) Problem IX in section 2. The associated minimax adjustment problem is similarly solvable; we omit details.

\subsection{Componentwise Rankings and Subvectors}

In section 4 we used the notation $\mathbf{x}_{j}$ for the subvector of $\mathbf{x}$ with components $\left\{x_{i}: i \epsilon S_{j}\right\}$. Let $\sigma(j)=\left|S_{j}\right|$ and let $\left\{x_{r}\right\}_{r=1}^{\sigma_{(j)}}$ be an enumeration of the components of $\mathbf{x}_{j}$. In this subsection we assume the "incomplete information" on $\mathbf{x}$, apart from the disaggregation conditions.

$$
s_{j}{ }^{-} \leqslant \mathbf{x}\left(S_{j}\right) \leqslant s_{j}^{+}
$$

and the ubiquitous

$$
\Sigma_{i} x_{i}=1,
$$

to be a prescribed rank order within each subvector,

$$
x_{1}^{j} \leqslant x_{z^{j}} \leqslant \ldots \leqslant x_{\sigma(j)^{j}} .
$$

We begin by determining the $M_{i}$ and $m_{i}$. If $x_{i}=x_{r}{ }^{j}$, write $w_{r}{ }^{j}, M_{r}{ }^{j}$ and $m_{r}{ }^{j}$ for $w_{i}, M_{i}$ and $m_{i}$. The analysis proceeds in two steps.

First, assume the vector $\mathbf{s}$ with $s_{j}=\mathbf{x}\left(S_{j}\right)$ is known. Then for each $j \epsilon\{1,2, \ldots, m\}$, a rescaling by $1 / s_{j}$ converts the problem into that leading to the previous equations (3.2.2-4). Thus, using a functional notation to express dependence on $s_{j}$, we have

$$
\begin{gathered}
M_{r}{ }^{j}\left(s_{j}\right)=s_{j} /[\sigma(j)+1-r] . \\
m_{r}{ }^{j}\left(s_{j}\right)=0 \quad \text { if } r<\sigma(j), \\
m_{\sigma(j)^{j}}\left(s_{j}\right)=s_{j} / \sigma(j) .
\end{gathered}
$$

Second, we take into account the possible range of variation of $s_{j}$, constrained as it is by

$$
\begin{gathered}
s_{j}{ }^{-} \leqslant s_{j} \leqslant s_{j}{ }^{+} \\
\Sigma_{j} s_{j}=1 .
\end{gathered}
$$

Determining this range is precisely the problem solved earlier to yield eqs. (3.1.13-14). Thus we obtain

$$
\begin{gathered}
M_{r}^{J}=\min \left\{s_{J^{+}}, 1-\Sigma_{j \neq J} s_{j}^{-}\right\} /[\sigma(J)+1-r], \\
m_{r}{ }^{J}=0 \quad \text { if } r<\sigma(J), \\
m_{\sigma(J)}{ }^{J}=\max \left\{s_{J^{-}}, 1-\Sigma_{j \neq J} s_{j}^{+}\right\} / \sigma(J) .
\end{gathered}
$$


For the main analysis, we wish to determine the least $z \geqslant 0$ for which an $\mathbf{x}$ exists satisfying (5.2.1-3) as well as

$$
\max \left\{0, M_{i}-z / w_{i}\right\} \leqslant x_{i} \leqslant m_{i}+z / w_{i}
$$

Again we proceed in two steps, first treating all $s_{j}=\mathbf{x}\left(S_{j}\right)$ as known. Thus for all $j$, we seek a subvector $\mathbf{x}_{j}$ which satisfies (5.2.3) as well as

$$
\max \left\{0, M_{r^{j}}-z / w_{r^{j}}\right\} \leqslant x_{r^{j}} \leqslant m_{r}{ }^{j}+z / w_{r^{j}}
$$

for $1 \leqslant r \leqslant \sigma(j)$, and

$$
\sum_{r=1}^{\sigma(j)} x_{r}{ }^{j}=s_{j}
$$

This is an instance of Problem II in section 2; the conditions for solutions $\mathbf{x}_{j}$ to exist read

$$
\begin{gathered}
\max \left\{0, M_{r}{ }^{j}-z / w_{r}\right\} \leqslant m_{R}^{j}+z / w_{R}^{j} \quad(r \leqslant R) \\
\sum_{r=1}^{\sigma(j)} \max _{R \leqslant r}\left\{0, M_{R}{ }^{j}-Z / w_{R}^{j}\right\} \leqslant s_{j}, \\
\sum_{r=1}^{\sigma(j)} \min _{R \geqslant r}\left(m_{R}^{j}+z / w_{R}^{j}\right) \geqslant s_{j} .
\end{gathered}
$$

Here (5.2.11) does not involve $s_{j}$; the least value of $z$ which satisfies it is readily found via

$$
\begin{gathered}
z_{j}^{\circ}=\max _{1 \leqslant r \leqslant R \leqslant \sigma(j)}\left\{\left(M_{r^{j}}-m_{R^{j}}\right) /\left(1 / w_{r}{ }^{j}+1 / w_{R}{ }^{j}\right)\right\}, \\
z^{\circ}=\max \left\{0, \max _{j} z_{j}^{\circ}\right\} .
\end{gathered}
$$

Next, rewrite (5.2.12) as

$$
\sum_{r=1}^{\sigma(j)} \max \left\{0, \max _{R \leqslant r}\left(M_{R}^{j}-z / w_{R}^{j}\right)\right\} \leqslant s_{j}
$$

and repeat $(5.2 .13)$ as

$$
\sum_{r=1}^{\sigma(j)} \min _{R \geqslant r}\left(m_{R}^{j}+z / w_{R}^{j}\right) \geqslant s_{j} .
$$

The question of existence for a vector s obeying both (5.2.4-5) and (5.2.16-17) is an instance of Problem I in section 2; the conditions for existence are

$\max \left\{s_{j}^{-}, \sum_{r=1}^{\sigma(j)} \max \left\{0, \max _{R \leqslant r}\left(M_{R^{j}}-z / w_{R^{j}}\right)\right\}\right\} \leqslant \min \left\{s_{j}{ }^{+}, \sum_{r=1}^{\sigma(j)} \min _{R \geqslant r}\left(m_{R}{ }^{j}+z / w_{R}{ }^{j}\right)\right\}$

for all $j$, and

$$
\begin{gathered}
\sum_{j} \max \left\{s_{j}^{-}, \sum_{r=1}^{\sigma(j)} \max \left\{0, \max _{R \leqslant r}\left(M_{R}^{j}-z / w_{R}^{j}\right)\right\}\right\} \leqslant 1, \\
\Sigma_{j} \min \left\{s_{j}^{+}, \sum_{r=1}^{\sigma(j)} \min _{R \geqslant r}\left(m_{R}^{j}+z / w_{R^{j}}\right)\right\} \geqslant 1 .
\end{gathered}
$$

The new information deducible from (5.2.18) consists of the two inequalities

$$
\begin{gathered}
\sum_{r=1}^{\sigma(j)} \max \left\{0, \max _{R \leqslant r}\left(M_{R^{j}}-z / w_{R^{j}}\right)\right\} \leqslant s_{j}{ }^{+}, \\
\sum_{r=1}^{\sigma(j)} \min _{R \geqslant r}\left(m_{R}{ }^{j}+z / w_{R^{j}}\right) \geqslant s_{j}{ }^{-} .
\end{gathered}
$$

Finding the least $z \geqslant 0$ satisfying (5.2.21) is an instance of unsolved Problem V in section 2; similarly for (5.2.19) and unsolved Problem X. In view of (5.2.7), relation (5.2.22) can be rewritten

$$
\begin{gathered}
\left(m^{j}+z / w^{j}\right)+\Sigma_{r<\sigma(j)} \min \left\{m^{j}+z / w^{j}, z / v_{r^{j}}\right\} \geqslant s_{j}^{-} \\
\left(m^{j}=m_{\sigma(j)^{j}}, w^{j}=w_{\sigma(j)^{j}}, v_{r}{ }^{j}=\max _{r \leqslant R<\sigma(j)} w_{R^{j}}\right),
\end{gathered}
$$


and the least $z \geqslant 0$ satisfying it can be found using the technique illustrated near (3.2.20-23) in the previous text. Finding the least $z \geqslant 0$ obeying (5.2.20) is a priori an instance of unsolved Problem XI, and appeal to (5.2.7) has not so far yielded reduction to a solved Problem despite the initial simplification to

$$
\Sigma_{j} \min \left\{s_{j^{+}}, m^{j}+z / w^{j}+\Sigma_{r<\sigma(j)} \min \left\{m^{j}+z / w^{j}, z / v_{r^{j}}\right\}\right\} \geqslant 1 .
$$

In view of these difficulties, we first specialize to the case

$$
\left\{w_{r}\right\}_{r=1}^{\sigma(j)} \text { nondecreasing, for all } j .
$$

Then (5.2.19) and (5.2.21) become

$$
\begin{gathered}
\sum_{j} \max \left\{s_{j}^{-}, \sum_{r=1}^{\sigma(j)} \max \left\{0, M_{r}{ }^{j}-z / w_{r}{ }^{j}\right\}\right\} \leqslant 1, \\
\sum_{r=1}^{\sigma(j)} \max \left\{0, \mathrm{M}_{r}{ }^{j}-z / w_{r}{ }^{j}\right\} \leqslant s_{j}{ }^{+},
\end{gathered}
$$

and so can be handled as instances of (solved) Problems IX and III respectively. The treatment of (5.2.23) simplifies as in the analysis leading to (3.2.26). Equation (5.2.14), or more directly (5.2.11), gives

$$
z_{j}^{\circ}=\frac{1}{2} w^{j}-\left(M^{j}-m^{j}\right) \quad\left(M^{j}=M_{\sigma(j)^{j}}\right) .
$$

However, (5.2.24) still appears recalcitrant despite the additional information $v_{r}{ }^{j} \leqslant w^{j}$.

We therefore retreat still further, to the semi-weighted case $\left(w_{i}=W_{j}\right.$ for all $\left.i \epsilon S_{j}\right)$. Now $(5.2 .23)$ admits explicit solution

$$
z \geqslant W_{j}\left[s_{j}^{-}-m^{j}\right] / \sigma(j) .
$$

Most important, the previous maverick (5.2.24) becomes equivalent to

$$
\Sigma_{j} \max \left\{0,\left(s_{j}^{+}-m^{j}\right)-\sigma(j) z / W_{j}\right\} \leqslant \Sigma_{j} s_{j}^{+}-1
$$

so that its treatment is an instance of (solved) Problem III.

We turn now to the minimax adjustment problem. Let $a_{i}=a_{r}{ }^{j}$ if $x_{i}=x_{r}{ }^{j}$, and again begin by treating $\mathbf{s}$ as if it were known. Then the constraints on the components of subvector $\mathbf{x}_{j}$ read

$$
\begin{gathered}
\max \left\{0, a_{r^{j}}-z / w_{r}{ }^{j}\right\} \leqslant x_{r}{ }^{j} \leqslant a_{r}{ }^{j}+z / w_{r}{ }^{j}, \\
\sum_{r=1}^{\sigma(j)} x_{r}{ }^{j}=s_{j}, \\
x_{1}{ }^{j} \leqslant x_{r^{j}} \leqslant \ldots \leqslant x_{\sigma(j)^{j}} \leqslant
\end{gathered}
$$

The conditions for such an $\mathbf{x}_{j}$ to exist are

$$
\begin{gathered}
\max \left\{0, \max _{R} \leqslant r\left(a_{R}^{j}-z / w_{R}^{j}\right)\right\} \leqslant \min _{R} \geqslant r\left(a_{R}^{j}+z / w_{R}^{j}\right), \\
\sum_{r=1}^{\sigma(j)} \max \left\{0, \max _{R \leqslant r}\left(a_{R}^{j}-z / w_{R}^{j}\right)\right\} \leqslant s_{j}, \\
\sum_{r=1}^{\sigma(j)} \min _{R \geqslant r}\left(a_{R}^{j}+z / w_{R}^{j}\right) \geqslant s_{j} .
\end{gathered}
$$

The least $z$ satisfying $(5.2 .30)$ is given by

$$
\left.z_{j}^{\circ}=\max _{r \leqslant R}\left\{\left(a_{r^{j}}-a_{R}\right)^{j}\right) /\left(1 / w_{r}^{j}+1 / w_{R^{j}}\right)\right\} .
$$

The conditions for the existence of an s satisfying (5.2.31-32) as well as (5.2.4-5) are

$$
\begin{gathered}
\max \left\{s_{j}^{-}, \Sigma_{r=1}^{\sigma(j)} \max \left\{0, \max _{R} \leqslant r\left(a_{R}^{j}-z / w_{R}^{j}\right)\right\}\right\} \leqslant \min \left\{s_{j}^{+}, \Sigma_{r=1}^{\sigma(j)} \min _{R \geqslant r}\left(a_{R}^{j}+z / w_{R}^{j}\right)\right\}, \\
\Sigma_{j} \max \left\{s_{j}^{-}, \sum_{r=1}^{\sigma(j)} \max \left\{0, \max _{R \leqslant r}\left(a_{R}^{j}-z / w_{R}^{j}\right)\right\}\right\} \leqslant 1,
\end{gathered}
$$




$$
\Sigma_{j} \min \left\{s_{j}^{+}, \Sigma_{r=1}^{\sigma(j)} \min _{R \geqslant r}\left(a_{R}{ }^{j}+z / w_{R}{ }^{j}\right)\right\} \geqslant 1 .
$$

The new information from (5.2.34) is given by

$$
\begin{gathered}
\sum_{r=1}^{\sigma(j)} \max \left\{0, \max _{R \leqslant r}\left(a_{R}^{j}-z / w_{R}^{j}\right)\right\} \leqslant s_{j}^{+}, \\
\sum_{r=1}^{\sigma(j)} \min _{R \geqslant r}\left(a_{R}^{j}+z / w_{R^{j}}\right) \geqslant s_{j}^{-} .
\end{gathered}
$$

Here we have instances of unsolved Problems V, VI, X and XI; again we pass to the semi-weighted case $w_{r}{ }^{j}=W_{j}$. It is convenient to define

$$
b_{r}^{j}=\max _{R \leqslant r} a_{R}^{j}, c_{r}{ }^{j}=\min _{R \geqslant r} a_{R}^{j} .
$$

Then (5.2.33) yields

$$
z_{j}^{\circ}=\frac{1}{2} W_{j} \max _{r}\left(b_{r}^{j}-c_{r}{ }^{j}\right) .
$$

Relation (5.2.37) becomes

$$
\sum_{r=1}^{\sigma(j)} \max \left\{0, b_{r}{ }^{j}-z / W_{j}\right\} \leqslant s_{j}^{+},
$$

so determining the least value $z_{j}^{*}$ of $z \geqslant 0$ which satisfies it is an instance of Problem III; the least value of $z$ which satisfies $(5.2 .38)$ is

$$
\left.z_{j}^{* *}=\left[s_{j}^{-}-\sum_{r=1}^{\sigma(j)} c_{r}{ }^{j}\right)\right] W_{j} / \sigma(j) .
$$

Continuing, (5.2.35) becomes

$$
\Sigma_{j} \max \left\{s_{j}^{-}-\Sigma_{r=1}^{\sigma(j)} \max \left\{0, b_{r^{j}}-z / W_{j}\right\}\right\} \leqslant 1,
$$

while (5.2.36) becomes equivalent to

$$
\Sigma_{j} \max \left\{0,\left[s_{j}^{+}-\Sigma_{r=1}^{\sigma(j)} c_{r}^{j}\right]-z \sigma(j) / W_{j}\right\} \leqslant \Sigma_{j} s_{j}^{+}-1 .
$$

Finding the least values of $z \geqslant 0$ which satisfy these $\left(z^{* * *}\right.$ and $\left.z^{* * * *}\right)$ are instances of Problems IX and III. Thus finding the appropriate $z_{\min }$ can again be regarded as a solved problem.

\subsection{Both Componentwise Bounds and Rankings}

In this subsection, we impose both the constraints of the previous two subsections. Thus, writing $L_{r}{ }^{j}$ and $U_{r}{ }^{j}$ for $L_{i} \geqslant 0$ and $U_{i} \leqslant 1$ if $x_{i}=x_{r}{ }^{j}$, our polyhedron $P$ in $\mathbf{x}$-space is defined by

$$
\begin{gathered}
L_{r}{ }^{j \leqslant x_{r}}{ }^{j} \leqslant U_{r}{ }^{j} \\
\sum_{r=1}^{\sigma(j)} x_{r}{ }^{j}=s_{j}, \\
x_{1}{ }^{j} \leqslant x_{2}{ }^{j} \leqslant \ldots \leqslant x_{\sigma(j)^{j}}, \\
s_{j}{ }^{-} \leqslant s_{j} \leqslant s_{j}^{+}, \\
\Sigma_{j} s_{j}=1 .
\end{gathered}
$$

Without loss of generality, it may be assumed that

$$
\begin{gathered}
\left\{L_{r}{ }^{j}\right\}_{r=1}^{\sigma(j)} \quad \text { and } \quad\left\{U_{r}{ }^{j}\right\}_{r=1}^{\sigma(j)} \text { are nondecreasing } \\
\mathbf{L}\left(S_{j}\right)=\Sigma_{r} L_{r}{ }^{j} \leqslant s_{j}^{-} \leqslant s_{j}^{+} \leqslant \Sigma_{r} U_{r}{ }^{j}=\mathbf{U}\left(S_{j}\right)
\end{gathered}
$$


We first determine the conditions for $P$ to be nonempty. For given $\mathbf{s}$, finding an $\mathbf{x}_{j}$ satisfying (5.3.1-3) is an instance of Problem II; using (5.3.6), the conditions for a solution to exist are

$$
\begin{gathered}
L_{r}{ }^{j} \leqslant U_{r^{j}}, \\
\Sigma_{r} L_{r}{ }^{j} \leqslant s_{j} \leqslant \Sigma_{r} U_{r}{ }^{j} .
\end{gathered}
$$

It will be assumed that (5.3.8) holds. In view of (5.3.7), (5.3.9) is already implied by (5.3.4) and so can be ignored. Thus the remaining condition for $P$ to be nonempty is the existence of an $\mathbf{s}$ satisfying (5.3.4) and (5.3.5); this is an instance of Problem I, and the only new condition introduced is

$$
\Sigma_{j} s_{j}{ }^{-} \leqslant 1 \leqslant \Sigma_{j} s_{j}{ }^{+} .
$$

Assume for the moment that the $M_{i}$ and $m_{i}$ appropriate to this polyhedron $P$ are known, and observe that

$$
\left\{M_{r}^{j}\right\}_{r=1}^{\sigma(j)} \quad \text { and } \quad\left\{m_{r}\right\}_{r=1}^{\sigma(j)} \text { are nondecreasing. }
$$

We wish to determine the least $z \geqslant 0$ for which an $\mathbf{x}$ and $\mathbf{s}$ exist satisfying (5.3.1-5) as well as

$$
\max \left\{0, M_{r^{j}}-z / w_{r^{j}}\right\} \leqslant x_{r^{j}} \leqslant m_{r^{j}}+z / w_{r^{j}} .
$$

The conditions for the existence of an $\mathbf{x}_{j}$ satisfying (5.3.12) as well as (5.3.1-3) are

$$
\begin{gathered}
\max \left\{L_{r^{j}}, M_{r^{j}}-z / w_{r^{j}}\right\} \leqslant \min \left\{U_{R^{j}}, m_{R}^{j}+z / w_{R}^{j}\right\} \quad(r \leqslant R), \\
\sum_{r=1}^{\sigma(j)} \max _{R \leqslant r} \max \left\{L_{R}^{j}, M_{R}^{j}-z / w_{R}^{j}\right\} \leqslant s_{j}, \\
\sum_{r=1}^{\sigma(j)} \min _{R \geqslant r} \min \left\{U_{R}^{j}, m_{R}^{j}+z / w_{R}^{j}\right\} \geqslant s_{j} .
\end{gathered}
$$

The least $z \geqslant 0$ satisfying (5.3.13) is given, as in (5.2.14), by

$$
z_{j}^{\circ}=\max \left\{0, \max _{1 \leqslant r \leqslant R \leqslant \sigma(j)}\left\{\left(M_{r^{j}}-m_{R^{j}}\right) /\left(1 / w_{r}{ }^{j}+1 / w_{R}{ }^{j}\right)\right\}\right\} .
$$

Next, by Problem I, an s satisfying (5.3.4-5) as well as (5.3.14-15) exists if and only if

$$
\begin{gathered}
\sum_{r=1}^{\sigma(j)} \max _{R \leqslant r} \max \left\{L_{R^{j}}, M_{R} j-z / w_{R}^{j}\right\} \leqslant s_{j}^{+}, \\
\sum_{r=1}^{\sigma(j)} \min _{R \geqslant r} \min \left\{U_{R^{j}}, m_{R^{j}}+z / w_{R}^{j}\right\} \geqslant s_{j}^{-}, \\
\sum_{j} \max \left\{s_{j}^{-}, \sum_{r=1}^{\sigma(j)} \max _{R \leqslant r} \max \left\{L_{R^{j}}, M_{R}^{j}-z / w_{R^{j}}\right\}\right\} \leqslant 1, \\
\Sigma_{j} \min \left\{s_{j}^{+}, \sum_{r=1}^{\sigma(j)} \min _{R \geqslant r} \min \left\{U_{R^{j}}, m_{R^{j}}+z / w_{R^{j}}\right\}\right\} \geqslant 1 .
\end{gathered}
$$

The last two relations can be rewritten, using (5.3.6), as

$$
\begin{gathered}
\Sigma_{j} \max \left\{s_{j}{ }^{-}, \sum_{r=1}^{\sigma(j)} \max \left\{L_{r}{ }^{j}, \max _{R \leqslant r}\left(\dot{M}_{R}{ }^{j}-z / w_{R}{ }^{j}\right)\right\}\right\} \leqslant 1, \\
\Sigma_{j} \min \left\{s_{j}^{+}, \sum_{r=1}^{\sigma(j)} \min \left\{U_{r}{ }^{j}, \min _{R \geqslant r}\left(m_{R}{ }^{j}+z / w_{R}^{j}\right)\right\} \geqslant 1 .\right.
\end{gathered}
$$

These in turn can be rewritten

$$
\begin{gathered}
\Sigma_{j} \max \left\{s_{j}^{-}-\mathbf{L}\left(S_{j}\right), \Sigma_{r=1}^{\sigma(j)} \max \left\{0, \max _{R \leqslant r}\left(M_{R}{ }^{j}-L_{r}{ }^{j}-z / w_{R}{ }^{j}\right)\right\}\right\} \leqslant 1-\Sigma_{i} L_{i}, \\
\Sigma_{j} \max \left\{\mathbf{U}\left(S_{j}\right)-s_{j}{ }^{+}, \sum_{r=1}^{\sigma(j)} \max \left\{0, \max _{R \geqslant r}\left(U_{R^{j}}-z / w_{R^{j}}\right)\right\}\right\} \leqslant \Sigma_{i} U_{i}-1 .
\end{gathered}
$$

Since (5.3.17) can be written

$$
\sum_{r=1}^{\sigma(j)} \max \left\{0, \max _{R \leqslant r}\left(M_{R}{ }^{j}-L_{r}{ }^{j}-z / w_{R}{ }^{j}\right)\right\} \leqslant s_{j}{ }^{+}-\mathbf{L}\left(S_{j}\right),
$$


finding the least $z \geqslant 0$ which satisfies it is an instance of (unsolved) Problem $V$; the same is true for (5.3.18), which can be rewritten

$$
\sum_{r=1}^{\sigma(j)} \max \left\{0, \max _{R \geqslant r}\left(U_{r}^{j}-m_{R}^{j}-z / w_{R}^{j}\right)\right\} \leqslant \mathbf{U}\left(S_{j}\right)-s_{j}{ }^{-} .
$$

Finding the least $z \geqslant 0$ which satisfies (5.3.19) and (5.3.20) is an instance of (unsolved) Problem X.

In view of these difficulties we again withdraw to the semi-weighted case $\left(w_{r}{ }^{j}=W_{j}\right)$. Then (5.3.16) becomes

$$
z_{j}^{\circ}=\frac{1}{2} W_{j} \max _{r}\left(M_{r}^{j}-m_{r}^{j}\right) .
$$

(5.3.21) and (5.3.22) become

$$
\begin{aligned}
& \sum_{r=1}^{\sigma(j)} \max \left\{0,\left(M_{r}{ }^{j}-L_{r}{ }^{j}-z / W_{j}\right)\right\} \leqslant s_{j}{ }^{+}-\mathbf{L}\left(S_{j}\right), \\
& \sum_{r=1}^{\sigma(j)} \max \left\{0,\left(U_{r}{ }^{j}-m_{r}{ }^{j}\right)-z / W_{j}\right\} \leqslant \mathbf{U}\left(S_{j}\right)-s_{j}{ }^{-},
\end{aligned}
$$

so that finding the least $z \geqslant 0$ (call it $z_{j}^{*}$ ) satisfying (5.3.24), or the least $z \geqslant 0$ (call it $z_{j}^{* *}$ ) satisfying (5.3.25), is an instance of Problem III. Similarly, (5.3.19) and (5.3.20) become

$$
\begin{aligned}
& \Sigma_{j} \max \left\{s_{j}^{-}-\mathbf{L}\left(s_{j}\right), \Sigma_{r=1}^{\sigma(j)} \max \left\{0,\left(M_{r^{j}}-L_{r}{ }^{j}\right)-z / W_{j}\right\}\right\} \leqslant 1-\Sigma_{i} L_{i}, \\
& \Sigma_{j} \max \left\{\mathbf{U}\left(S_{j}\right)-s_{j}{ }^{+}, \Sigma_{r=1}^{\sigma(j)} \max \left\{0,\left(U_{r^{j}}-m_{r}{ }^{j}\right)-z / W_{j}\right\}\right\} \leqslant \Sigma_{i} U_{i}-1 .
\end{aligned}
$$

Finding the least $z \geqslant 0$ (call it $z^{* * *}$ ) which satisfies (5.3.26), or the least $z \geqslant 0$ (call it $z^{* * * *}$ ) which satisfies (5.3.27), is an instance of Problem IX. Thus, determining the desired

$$
z_{\min }=\max \left\{\max _{j}\left(z_{j}^{\circ}, z_{j}^{*}, z_{j}^{* *}\right\}, z^{* * *}, z^{* * * *}\right\}
$$

can be regarded as a solved problem, given the $M_{i}$ and $m_{i}$.

We turn now to the associated minimax adjustment problem. First, finding an $\mathbf{x}_{j}$ which satisfies

$$
a_{r}{ }^{j}-z / w_{r}{ }^{j} \leqslant x_{r}{ }^{j} \leqslant a_{r}{ }^{j}+z / w_{r}{ }^{j}
$$

in addition to (5.3.1-3) is an instance of Problem II; a solution exists if and only if

$$
\begin{gathered}
\max \left\{L_{r}{ }^{j}, a_{r^{j}}-z / w_{r^{j}}\right\} \leqslant \min \left\{U_{R^{j}}, a_{R}{ }^{j}+z / w_{R}{ }^{j}\right\} \quad(r \leqslant R), \\
\sum_{r=1}^{\sigma(j)} \max _{R \leqslant r} \max \left\{L_{R}{ }^{j}, a_{R}{ }^{j}-z / w_{R}{ }^{j}\right\} \leqslant s_{j} \\
\sum_{r=1}^{\sigma(j)} \min _{R \geqslant r} \min \left\{U_{R}{ }^{j}, a_{R}{ }^{j}+z / w_{R}{ }^{j}\right\} \geqslant s_{j} .
\end{gathered}
$$

The least $z \geqslant 0$ satisfying (5.3.28) is given by

$$
z_{j}^{\circ}=\max \left\{0, z_{j}^{\circ \circ}, z_{j}^{\circ \circ}\right\}
$$

where

$$
\begin{gathered}
z_{j}^{\circ \circ}=\max _{r \leqslant R}\left[\left(a_{r}{ }^{j}-a_{R^{j}}\right) /\left(1 / w_{r}{ }^{j}+1 / w_{R}{ }^{j}\right)\right], \\
z_{j}^{\circ \circ \circ}=\max _{r} w_{r}{ }^{j} \max \left(L_{r}{ }^{j}-a_{r}{ }^{j}, a_{r}{ }^{j}-U_{r^{j}}\right) .
\end{gathered}
$$

Next, an s satisfying both (5.3.4-5) and (5.3.29-30) exists if and only if

$$
\left.\begin{array}{l}
\sum_{r=1}^{\sigma(j)} \max _{R} \leqslant r \max \left\{L_{R}{ }^{j}, a_{R}{ }^{j}-z / w_{R^{j}}\right\} \leqslant s_{j}{ }^{+} \\
\sum_{r=1}^{\sigma(j)} \min _{R} \geqslant r \min \left\{U_{R}{ }^{j}, a_{R}{ }^{j}+z / w_{R}{ }^{j}\right\} \geqslant s_{j}{ }^{-} \\
\Sigma_{j} \max \left\{s_{j}{ }^{-}, \sum_{r=1}^{\sigma(j)} \max _{R \leqslant r} \max \left\{L_{R}{ }^{j}, a_{R}{ }^{j}-z / w_{R}{ }^{j}\right\}\right\} \leqslant 1, \\
\Sigma_{j} \min \left\{s_{j}{ }^{+}, \sum_{r=1}^{\sigma(j)} \min _{R \geqslant r} \min \left\{U_{R^{j}}{ }^{j}, a_{R}{ }^{j}+a / w_{R}{ }^{j}\right\}\right\} \geqslant 1 .
\end{array}\right\}
$$


These four relations can be rewritten, respectively, as

$$
\begin{gathered}
\sum_{r=1}^{\sigma(j)} \max \left\{0, \max _{R \leqslant r}\left(a_{R^{j}}-L_{r^{j}}-z / w_{R^{j}}\right)\right\} \leqslant s_{j}^{+}-\mathbf{L}\left(S_{j}\right), \\
\sum_{r=1}^{\sigma(j)} \max \left\{0, \max _{R \geqslant r}\left(U_{r^{j}}-a_{R^{j}}-z / w_{R^{j}}\right)\right\} \leqslant \mathbf{U}\left(S_{j}\right)-s_{j}^{-}, \\
\Sigma_{j} \max \left\{s_{j}^{-}-\mathbf{L}\left(S_{j}\right), \sum_{r=1}^{\sigma(j)} \max \left\{0, \max _{R \leqslant r}\left(a_{R^{j}}-L_{r}{ }^{j}-z / w_{R^{j}}\right)\right\}\right\} \leqslant 1-\Sigma_{i} L_{i}, \\
\Sigma_{j} \max \left\{\mathbf{U}\left(S_{j}\right)-s_{j}^{+}, \sum_{r=1}^{\sigma(j)} \max \left\{0, \max _{R \geqslant r}\left(U_{r^{j}}-a_{R^{j}}-z / w_{R^{j}}\right)\right\}\right\} \leqslant \Sigma_{i} U_{i}-1 .
\end{gathered}
$$

Finding the least $z \geqslant 0$ which satisfies (5.3.35) or (5.3.36) is an instance of unsolved Problem V; finding the least $z \geqslant 0$ which satisfies (5.3.37) or (5.3.38) is an instance of unsolved Problem X. Thus we revert to the semi-weighted case. Then (5.3.31) yields

$$
z_{j}^{\circ \circ}=\frac{1}{2} W_{j} \max _{r}\left(b_{r}^{j}-c_{r}{ }^{j}\right),
$$

where $b_{r}{ }^{j}$ and $c_{r}{ }^{j}$ are defined in (5.2.39).

Relations (5.3.35-38) become

$$
\begin{gathered}
\sum_{r=1}^{\sigma(j)} \max \left\{0,\left(b_{r^{j}}-L_{r}{ }^{j}\right)-z / W_{j}\right\} \leqslant s_{j}{ }^{+}-\mathbf{L}\left(S_{j}\right), \\
\sum_{r=1}^{\sigma(j)} \max \left\{0,\left(U_{r^{j}}-c_{r^{j}}\right)-z / W_{j}\right\} \leqslant \mathbf{U}\left(S_{j}\right)-s_{j}^{-}, \\
\Sigma_{j} \max \left\{s_{j}^{-}-\mathbf{L}\left(S_{j}\right), \sum_{r=1}^{\sigma(j)} \max \left\{0,\left(b_{r^{j}}-L_{r^{j}}\right)-z / W_{j}\right\}\right\} \leqslant 1-\Sigma_{i} L_{i}, \\
\Sigma_{j} \max \left\{\mathbf{U}\left(S_{j}\right)-s_{j}{ }^{+}, \sum_{r=1}^{\sigma(j)} \max \left\{0, U_{r^{j}}-c_{r^{j}}-z / W_{j}\right\}\right\} \leqslant \Sigma_{i} U_{i}-1 .
\end{gathered}
$$

Finding the least value of $z \geqslant 0$ (called $z_{j}^{*}, z_{j}^{* *}, z^{* * *}, z^{* * * *}$ respectively) which satisfies each of these is an instance of either Problem III or Problem IX. Thus the determination of the desired

$$
z_{\min }=\max \left\{\max _{j}\left\{z_{j}^{\circ \circ}, z_{j}^{\circ \circ \circ}, z_{j}^{*}, z_{j}^{* *}\right\}, z^{* * *}, z^{* * * *}\right\}
$$

can be regarded as a solved problem.

We return now to determining the $M_{i}$ and $m_{i}$, i.e., the $M_{r}{ }^{j}$ and $m_{r}{ }^{j}$, for the polyhedron $P$ in $\mathbf{x}$ space defined by (5.3.1-5). Consider a fixed variable $x_{i}=x_{r}{ }^{j}$. Regarding $s_{j}$ as given, we can find an $M_{r}{ }^{j}\left(s_{j}\right)$ and $m_{r}{ }^{j}\left(s_{j}\right)$ for the polyhedron $P_{j}$ in $\mathbf{x}_{j}$-space defined by (5.3.1-3). Examining the analysis at the beginning of subsection 3.3 , we find (cf. (3.3.13-15)) that

$$
\begin{aligned}
& M_{r}{ }^{j}\left(s_{j}\right)=\min \left\{U_{r^{j}}, p_{r}{ }^{j}\left(s_{j}\right)\right\}, \\
& m_{r}{ }^{j}\left(s_{j}\right)=\max \left\{L_{r}{ }^{j}, q_{r}{ }^{j}\left(s_{j}\right)\right\},
\end{aligned}
$$

where $p r^{j}\left(s_{j}\right)$ is the unique $p$ such that

$$
p+\Sigma_{R \geqslant r} \max \left\{L_{R}^{j}, p\right\}=s_{j}-\Sigma_{R<r} L_{R}^{j}
$$

and $q_{r}{ }^{j}\left(s_{j}\right)$ is the unique $q$ such that

$$
q+\Sigma_{R<r} \min \left\{U_{R}^{j}, q\right\}=s_{j}{ }^{-} \Sigma_{R \geqslant r} U_{R}^{j} .
$$

As noted in subsection 3.3, these equations can be transformed, for any given $s_{j}$, into instances of (solved) Problem IV. Clearly $p_{r}{ }^{j}\left(s_{j}\right)$ and $q_{r}{ }^{j}\left(s_{j}\right)$ are increasing functions of $s_{j}$; if $t_{j}{ }^{+}$and $t_{j}{ }^{-}$denote the maximum and minimum possible values of $s_{j}$, then

$$
\begin{gathered}
M_{r}{ }^{j}=\min \left\{U_{r}{ }^{j}, p_{j}^{r}\left(t_{j}^{+}\right)\right\}, \\
m_{r}{ }^{j}=\max \left\{L_{r}{ }^{j}, q_{j}^{r}\left(t_{j}^{-}\right)\right\} .
\end{gathered}
$$

Finally, the determination of $t_{j}^{+}$and $t_{j}^{-}$is an instance of the problem leading to (3.1.13-14); the results are 


$$
\begin{gathered}
t_{j}^{+}=\min \left\{s_{j}^{+}, 1-\Sigma_{J \neq j} s_{J}^{-}\right\}, \\
t_{j}^{-}=\max \left\{s_{j}^{-}, 1-\Sigma_{J \neq j} s_{J}^{+}\right\} .
\end{gathered}
$$

\subsection{Mixed Componentwise Bounds and Rankings}

In this section, we consider the case in which some components $s_{j}$ of the "fuzzy" distribution $\mathbf{s}$ have their disaggregation constrained by upper and lower bounds, while the disaggregations of the remaining components are subject to ranking conditions. Specifically, there is a partition

$$
\{1,2, \ldots, m\}=B \cup Q
$$

into nonempty sets, and for convenience we define

$$
I=\cup\left\{S_{j}: j \epsilon B\right\} .
$$

The relevant polyhedron $P$ in $\mathbf{x}$-space is defined by

$$
\begin{gathered}
0 \leqslant x_{1}{ }^{j} \leqslant x_{2}{ }^{j} \leqslant \ldots x_{\sigma(j)}{ }^{j} \\
L_{i} \leqslant x_{i} \leqslant U_{i} \quad(i \epsilon I), \\
\sum_{i \epsilon S_{j}} x_{i}=s_{j}, \\
s_{j}{ }^{-} \leqslant s_{j} \leqslant s_{j}{ }^{+}, \\
\Sigma_{j S j}=1 .
\end{gathered}
$$

It will be assumed that

$$
\begin{array}{cc}
0 \leqslant L_{i} \leqslant U_{i} & \text { for } i \epsilon I, \\
\mathbf{L}\left(S_{j}\right) \leqslant s_{j}^{-} \leqslant s_{j}^{+} \leqslant \mathbf{U}\left(S_{j}\right) & \text { for } j \epsilon B, \\
\Sigma_{j} s_{j}^{-} \leqslant 1 \leqslant \Sigma_{j} s_{j}^{+} . &
\end{array}
$$

We first observe that for any s satisfying (5.4.4-5), there exists an $\mathbf{x}$ satisfying (5.4.1-3). Indeed, for each $j \epsilon Q$ there is clearly an $\mathbf{x}_{j}$ obeying (5.4.1) and (5.4.3); for each $j \epsilon B$ the existence of an $\mathbf{x}_{j}$ satisfying (5.4.2) and (5.4.3) is an instance of Problem I, and the associated conditions

$$
L_{i} \leqslant U_{i}, \mathbf{L}\left(S_{j}\right) \leqslant s_{j} \leqslant \mathbf{U}\left(S_{j}\right)
$$

are indeed satisfied, by virtue of (5.4.6-7).

Next we determine the $M_{i}$ and $m_{i}$. First, for each $j \epsilon Q$, the corresponding $M_{r}{ }^{j}$ and $m_{r}{ }^{j}$ are as in subsection 5.2 (see (5.2.6-8)); this follows from the observation above. Next, for a fixed $j \epsilon B, i \epsilon S_{j}$, and $x_{i} \epsilon\left[L_{i}, U_{i}\right]$, consider Problem I applied to $\mathbf{x}_{j}$ with $S=s_{j}, A_{i}=B_{i}=x_{i}$, and

$$
A_{k}=L_{k}, B_{k}=U_{k} \quad \text { for } k \in S_{j}-\{i\} .
$$

The conditions for a solution are

$$
x_{i}+\Sigma_{k \neq i} L_{k} \leqslant s_{j} \leqslant x_{i}+\Sigma_{k \neq i} U_{i},
$$

so that (with $S(j)=S_{j}$ )

$$
\begin{aligned}
& M_{i}\left(s_{j}\right)=\min \left\{U_{i}, s_{j}-\Sigma_{k \epsilon S(j)-\{i\}} L_{k}\right\}, \\
& m_{i}\left(s_{j}\right)=\max \left\{L_{i}, s_{j}-\Sigma_{k \epsilon S(j)-\{i\}} U_{k}\right\},
\end{aligned}
$$

for $i \epsilon S_{j}, j \epsilon B$. Since $M_{i}\left(s_{j}\right)$ and $m_{i}\left(s_{j}\right)$ are nondecreasing in $s_{j}, M_{i}$ is obtained as $M_{i}\left(t_{j}{ }^{+}\right)$and $m_{i}$ as $m_{i}\left(t_{j}{ }^{-}\right)$ 
where $t_{j}{ }^{+}, t_{j}{ }^{-}$are the maximum and minimum possible values of $s_{j}$, given in (5.2.49-50). Thus

$$
\begin{aligned}
& M_{i}=\min \left\{U_{i}, s_{j}^{+}-\Sigma_{k \epsilon S(j)-\{i\}} L_{k}, 1-\Sigma_{J \neq j} s_{j}^{-}-\Sigma_{k \epsilon S(j)-\{i\}} L_{k}\right\}, \\
& m_{i}=\max \left\{L_{i}, s_{j}^{-}-\Sigma_{k \epsilon S(j)-\{i\}} U_{k}, 1-\Sigma_{J \neq j} S J^{+}-\Sigma_{k \epsilon S(j)-\{i\}} U_{k}\right\} .
\end{aligned}
$$

The minimax selection problem will now be taken up. For each $j \epsilon B$, the conditions on $\mathbf{x}_{j}$ are

$$
\begin{gathered}
\max \left\{L_{i}, M_{i}-z / w_{i}\right\} \leqslant x_{i} \leqslant \min \left\{U_{i}, m_{i}+z / w_{i}\right\} \quad\left(i \epsilon S_{j}\right), \\
\sum_{i \epsilon S(j)} x_{i}=s_{j} .
\end{gathered}
$$

These conditions can be met, for $z \geqslant 0$, if and only if

$$
\begin{array}{ll}
z \geqslant z_{j}{ }^{\circ}=\frac{1}{2} \max _{i \epsilon S_{j}} w_{i}\left(M_{i}-m_{i}\right) & (j \epsilon B), \\
\sum_{i \epsilon S_{j}} \max \left\{L_{i}, M_{i}-z / w_{i}\right\} \leqslant s_{j} & (j \epsilon B), \\
\sum_{i \epsilon S_{j}} \min \left\{U_{i}, m_{i}+z / w_{i}\right\} \geqslant s_{j} & (j \epsilon B) .
\end{array}
$$

For each $j \epsilon Q$, the conditions on $\mathbf{x}_{j}$ are (5.4.1), (5.4.3), and

$$
\max \left\{0, M_{r}{ }^{j}-z / w_{r}{ }^{j}\right\} \leqslant x_{r}{ }^{j} \leqslant m_{r}{ }^{j}+z / w_{r}{ }^{j} .
$$

These conditions can be met, for $z \geqslant 0$, if and only if

$$
\begin{gathered}
\max \left\{0, M_{r^{j}}-z / w_{r^{j}}\right\} \leqslant m_{R}{ }^{j}+z / w_{R^{j}} \quad(r \leqslant R), \\
\sum_{r=1}^{\sigma(j)} \max _{R \leqslant r} \max \left\{0, M_{R}{ }^{j}-z / w_{R}{ }^{j}\right\} \leqslant s_{j} \quad(j \in Q), \\
\sum_{r=1}^{\sigma(j)} \min _{R \geqslant r}\left(m_{R}{ }^{j}+z / w_{R}{ }^{j}\right) \geqslant s_{j} \quad(j \in Q) .
\end{gathered}
$$

The new information from (5.4.16) is

$$
z \geqslant z_{j}^{\circ \circ}=\max _{r \leqslant R}\left[\left(M_{r^{j}}-m_{R^{j}}\right) /\left(1 / w_{r}{ }^{j}+1 / w_{R}{ }^{j}\right)\right] .
$$

Next, an s satisfying (5.4.4-5), (5.4.14-15) and (5.4.17-18) can exist if and only if

$$
\begin{aligned}
& \Sigma_{i \epsilon S_{j}} \max \left\{L_{i}, M_{i}-z / w_{i}\right\} \leqslant s_{j}^{+} \quad(j \in B), \\
& \Sigma_{i \epsilon S_{j}} \min \left\{U_{i}, m_{i}+z / w_{i}\right\} \geqslant s_{j}^{-} \quad(j \in B), \\
& \sum_{r=1}^{\sigma(j)} \max _{R \leqslant r} \max \left\{0, M_{R^{j}}-z / w_{R}{ }^{j}\right\} \leqslant s_{j}{ }^{+} \quad(j \in Q), \\
& \sum_{r=1}^{\sigma(j)} \min _{R \geqslant r}\left(m_{R}{ }^{j}+z / w_{R^{j}}{ }^{j}\right) \geqslant s_{j}{ }^{-} \quad(j \in Q), \\
& \Sigma_{j \in B} \max \left\{s_{j}^{-}, \Sigma_{i \epsilon S_{j}} \max \left\{L_{i}, M_{i}-z / w_{i}\right\}\right\} \\
& +\Sigma_{j \in Q} \max \left\{s_{j}^{-}, \sum_{r=1}^{\sigma(j)} \max _{R \leqslant r} \max \left\{0, M_{R^{j}}{ }^{j}-z / w_{R^{j}}{ }^{j}\right\}\right\} \leqslant 1, \\
& \sum_{j \in B} \min \left\{s_{j}{ }^{+}, \Sigma_{i \epsilon S_{j}} \min \left\{U_{i}, m_{i}+z / w_{i}\right\}\right\} \\
& +\sum_{j \in Q} \min \left\{s_{j}^{+}, \sum_{r=1}^{\sigma(j)} \min _{R \geqslant r}\left(m_{R}{ }^{j}+z / w_{R}{ }^{j}\right)\right\} \geqslant 1 .
\end{aligned}
$$

For $j \epsilon B$, let $z_{j}^{*}$ and $z_{j}^{* *}$ be the least values of $z \geqslant 0$ satisfying (5.4.20) and (5.4.21) respectively. Writing these relations as 


$$
\begin{aligned}
& \sum_{i \epsilon S_{j}} \max \left\{0,\left(M_{i}-L_{i}\right)-z / w_{i}\right\} \leqslant s_{j}{ }^{+}-\mathbf{L}\left(S_{j}\right), \\
& \sum_{i \epsilon S_{j}} \max \left\{0,\left(U_{i}-m_{i}\right)-z / w_{i}\right\} \leqslant \mathbf{U}\left(S_{j}\right)-s_{j}{ }^{-},
\end{aligned}
$$

we see that finding $z_{j}^{*}$ and $z_{j}^{* *}$ are instances of Problem III.

For $j \in R$, let $z_{j}^{*}$ and $z_{j}^{* *}$ be the least values of $z \geqslant 0$ satisfying (5.4.22) and (5.4.23) respectively. Their determination is an instance of unsolved Problem V or VI respectively, and so we now specialize to the case

$$
w_{i}=W_{j} \quad\left(i \epsilon S_{j}, j \epsilon Q\right) .
$$

Then (5.4.22) yields

$$
\sum_{r=1}^{\sigma(j)} \max \left\{0, M_{r}^{j}-z / W_{j}\right\} \leqslant s_{j}^{+},
$$

so that determining $z_{j}^{*}$ is an instance of Problem III. Relation (5.4.23) yields

$$
z_{j}^{* *}=\max \left\{0, W_{j}\left[s_{j}^{-}-\Sigma_{i \epsilon S_{j}} m_{i}\right] / \sigma(j)\right\} \quad(j \in Q)
$$

where the sum can be evaluated using (5.2.6-8). Relations (5.4.24) and (5.4.25) become equivalent to $\Sigma_{j \epsilon B} \max \left\{s_{j}^{-}-\mathbf{L}\left(S_{j}\right), \Sigma_{i \epsilon S_{j}} \max \left\{0, M_{i}-z / w_{i}\right\}\right\}$

$$
+\Sigma_{j \in Q} \max \left\{s_{j}^{-}, \Sigma_{i \epsilon S_{j}} \max \left\{0, M_{i}-z / W_{j}\right\}\right\} \leqslant 1-\Sigma_{i \epsilon I} L_{i},
$$

$\Sigma_{j \in B} \max \left\{\mathbf{U}\left(S_{j}\right)-s_{j}{ }^{+}, \Sigma_{i \epsilon S_{j}} \max \left\{0,\left(U_{i}-m_{i}\right)-z / w_{i}\right\}\right\}$

$$
+\Sigma_{j \in Q} \max \left\{0,\left(s_{j}{ }^{+}-\Sigma_{i \epsilon S_{j}} m_{i}\right)-z \sigma(j) / W_{j}\right\} \leqslant \Sigma_{i \epsilon I} U_{i}+\Sigma_{j \in Q} s_{j}{ }^{+}-1 .
$$

Let $z^{* * *}$ and $z^{* * * *}$ be the least values of $z \geqslant 0$ satisfying (5.4.30) and (5.4.31) respectively; then determining either of them is an instance of Problem IX. Thus finding the desired

$$
z_{\min }=\max \left\{\max _{j}\left\{z_{j}^{\circ}, z_{j}^{\circ}, z_{j}^{*}, z_{j}^{* *}\right\}, z^{* * *}, z^{* * * *}\right\}
$$

can be regarded as a solved problem.

The analysis of the associated minimax adjustment problem is analogous and will not be worked out here.

\section{Concluding Remarks}

The following table summarizes the status of the various topics treated. The material covered omits that of subsection 5.4 (which does not fall into the general pattern) and that of subsection 5.0 (subsumed by subsection 5.1). The status of the minimax selection and minimax adjustment problems is essentially the same in every case, so the two are not considered separately. "Ordinary" refers to the analyses in section 3, "disaggregation" to the topics in sections 4 and 5 . "Bottleneck" refers to the unsolved Problem (or Problems) in section 2 which stands in the way of a satisfactory solution method for the general weighted problem.

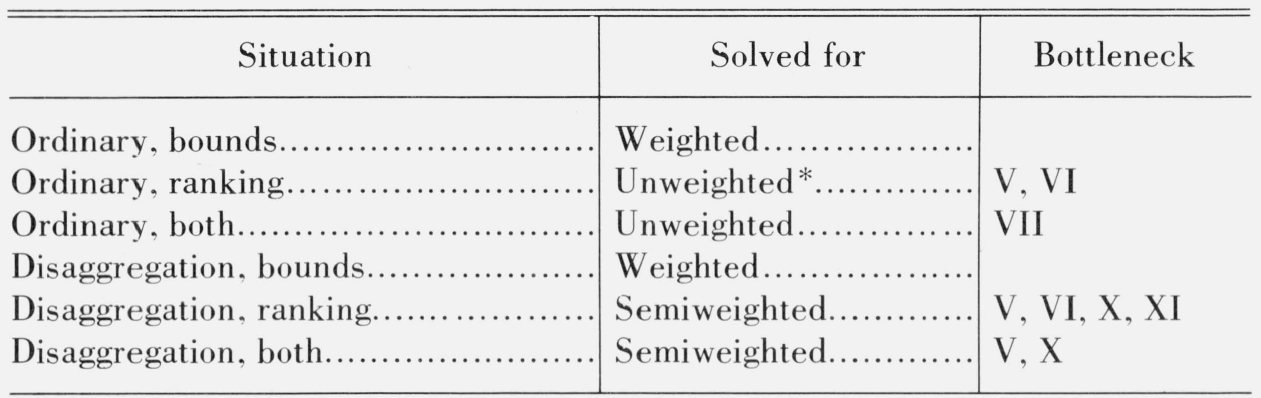

*The selection problem is also solved for $w_{n}=\max _{i} w_{i}$. 


\section{References}

[1] Goldman, A. J., and Meyers, P. R., Minimax error selection of a discrete univariate distribution with prescribed componentwise bounds, J. Res. Nat. Bur. Stand. (U.S.) $72 B$ (Math. Sci.), No. 4, 263-271 (1968).

[2] Goldman, A. J., Minimax error selection of a discrete univariate distribution with prescribed componentwise ranking, J. Res. Nat. Bur. Stand. (U.S.) 72B (Math. Sci.), No. 4, 273-277 (1968).

[3] Goldman, A. J., Minimax error selection of a univariate distribution with prescribed componentwise bounds and ranking, J.
Res. Nat. Bur. Stand. (U.S.) 73 B (Math. Sci.), No. 3, 225-230 (1969).

[4] Goldman, A. J., Minimax adjustment of a univariate distribution to satisfy componentwise bounds and/or ranking, J. Res. Nat. Bur. Stand. (U.S.) 73 B (Math. Sci.), No. 3, 231-239 (1969).

[5] Goldman, A. J., and Meyers, P. R., Minimax error selection of a bivariate distribution with prescribed marginals, (in preparation).

(Paper 76B1\&2-358) 